myjournal manuscript No.

(will be inserted by the editor)

\title{
Constraint Solving for Finite Model Finding in SMT Solvers
}

\author{
Andrew Reynolds · Cesare Tinelli · Clark \\ Barrett
}

Received: date / Accepted: date

\begin{abstract}
SMT solvers have been used successfully as reasoning engines for automated verification and other applications based on automated reasoning. Current techniques for dealing with quantified formulas in SMT are generally incomplete, forcing SMT solvers to report "unknown" when they fail to prove the unsatisfiability of a formula with quantifiers. This inability to return counter-models limits their usefulness in applications that produce queries involving quantified formulas. In this paper, we reduce these limitations by integrating finite model finding techniques based on constraint solving into the architecture used by modern SMT solvers. This approach is made possible by a novel solver for cardinality constraints, as well as techniques for on-demand instantiation of quantified formulas. Experiments show that our approach is competitive with the state of the art in SMT, and orthogonal to approaches in automated theorem proving.
\end{abstract}

Keywords Satisfiability Modulo Theories · Finite Model Finding

Under consideration for publication in Theory and Practice of Logic Programming (TPLP)

\section{Introduction}

Satisfiability modulo theories (SMT) is a subfield of automated reasoning concerned with the problem of determining the satisfiability of formulas in some first-

The work of the first two authors was partially funded by a grant from Intel Corporation.

Andrew Reynolds

Department of Computer Science, The University of Iowa

E-mail: andrew.j.reynolds@gmail.com

Cesare Tinelli

Department of Computer Science, The University of Iowa

E-mail: cesare-tinelli@uiowa.edu

Clark Barrett

Department of Computer Science, Stanford University

E-mail: barrett@cs.stanford.edu 
order theory $T$, where $T$ is usually a combination of several sub-theories. SMT techniques and solvers have been used successfully in recent years to support a variety of formal methods for hardware and software development, including automated verification. They are especially effective for tasks that can be reduced to proving the unsatisfiability of quantifier-free formulas in certain theories, such as theories of linear arithmetic, algebraic datatypes, bit vectors, arrays, strings and so on, for which it is possible to build specialized constraint solvers. A number of applications, however, require reasoners that can prove the unsatisfiability of quantified formulas in those theories. In verification applications, for instance, quantified formulas are necessary to express properties of systems with an unbounded number of processes, or properties involving a number of memory locations. In general, the need for quantified formulas arises naturally when dealing with function or predicate symbols that do not belong to the signature of an SMT solver's built-in theories.

The few SMT solvers that can currently reason about quantified formulas are based on incomplete methods and often report "unknown" when they fail, after some predetermined amount of effort, to prove a quantified formula unsatisfiable. For many client applications, however, it is very useful to know if the failure is due to the fact that the input formula is indeed satisfiable, especially if the solver can also return some representation of the formula's model. Current SMT solvers are able to produce models of satisfiable quantified formulas only in fairly restricted cases [17, which limits their scope and usefulness.

We reduce these limitations with a novel approach for model finding in SMT. Since, by the undecidability of first-order logic, there are no automated methods for finding arbitrary models, we focus on finite models, which can be represented symbolically and enumerated. More precisely, because SMT solvers work with sorted logics with both built-in and free ("uninterpreted") sorts, we focus on finding models that interpret the free sorts as finite domains. As with traditional finite model finders for first-order logic, the main idea is simply to check universally quantified formulas exhaustively over candidate models with increasingly large domains for the free sorts, until an actual model is found. Our approach differs from previous ones in that it does not rely on the explicit introduction of domain constants for the free sorts, as done by MACE-style model finders [10], and in that we are able to reason modulo more theories than just the theory of equality, unlike SEM-style model finders 38. Moreover, and crucially for our goals, the approach is fully integrated into the general architecture underlying most SMT solvers.

While limited to SMT formulas with quantifiers ranging only over free sorts or built-in finite sorts (such as, for instance, bit vector sorts or enumeration sorts), our approach is still quite useful. Formulas with such quantifiers occur often, for instance, in verification applications; moreover, when they are satisfiable they usually have small finite models.

We present our model finding method in the context of an abstract framework that models a large class of SMT solvers supporting multiple theories and quantified formulas 23 . We incorporate in this framework an efficient mechanism for deciding the satisfiability of a set of ground SMT formulas under finite cardinality constraints for the free sorts. This is used first to find a candidate model, a model $\mathcal{M}$ of a heuristically generated finite set of ground consequences of the input formula $\varphi$. To check that $\mathcal{M}$ satisfies $\varphi$ as well, the model finder then verifies, in a complete way, that all ground consequences of $\varphi$ over the universe of $\mathcal{M}$ are 
satisfied by $\mathcal{M}$. When this check fails, the model finder looks for a new candidate model, possibly under extended cardinality bounds for the free sorts. The practical effectiveness of this approach relies on two crucial components: $(i)$ a method for constructing and representing candidate models efficiently and (ii) a model-based quantifier instantiation heuristic that avoids the explicit generation and checking of all the ground instances of the input formula. The two are strictly related since the instantiation heuristic takes advantage of the way candidate models are represented to identify, and ignore, entire sets of instances that do not need to be considered.

The paper is organized as follows. After discussing preliminaries in Section 3 , we present the framework used by SMT solvers in Section 4 . We then present a high-level overview of our approach for finite model finding in Section 5 followed by details in Sections 68 This includes, in particular, the strategy used by the solver for finding small candidate finite models and the algorithm for checking the satisfiability of quantified formulas against these candidate models. Section 9 describes an experimental evaluation of our implementation of these techniques in the SMT solver CVC4 on several sets of benchmarks.

This paper builds on material from previous conference papers [29]30], as well as the PhD dissertation by the first author 32 .

\section{Related Work}

Most traditional finite model finders for quantified formulas are based on a reduction to a decidable logic, propositional logic or some decidable fragments of first-order logic, where the reduction introduces finite upper bounds on the cardinalities of the atomic types. This technique was pioneered by McCune in the Mace tool [24], and is often referred to as MACE-style model finding. These techniques were later implemented in the tool Paradox [10, which incorporated successful techniques for symmetry breaking. Other conceptually similar tools include FMDarwin [6], which handles first-order logic with equality, the Alloy Analyzer with its backend Kodkod [36] which handles first-order relational logic, and Nitpick [8] which handles higher-order logic. Recently, a MACE-style finite model finding approach was also implemented in the Vampire theorem prover [28].

A different approach to model finding, pioneered by the SEM model finder [38], does not encode the input problem into propositional logic. Instead, it uses builtin support for equality together with constraint propagation techniques similar to those used in modern constraint solvers. In this respect, our approach is more similar to SEM-style model finding than it is to MACE-style model finding.

Our approach uses on-demand quantifier instantiation to check the satisfiability of universally quantified formulas. Other instantiation-based approaches have been developed, both in the automated theorem proving community [21] and in SMT. For the latter, instantiation-based techniques are most typically used in an incomplete way for finding proofs of unsatisfiability [13, 16, 25]. Other techniques establish the satisfiability of quantified formulas, either by using model-based techniques [17, or by reasoning in local theories where only a finite set of instances is required for completeness [19]. 


\section{Preliminaries}

We work in the context of many-sorted first-order logic with equality. A (manysorted) signature $\Sigma$ consists of a set of sort symbols and a set of (sorted) function symbols, $f: S_{1} \times \cdots \times S_{n} \rightarrow S$, where $n \geq 0$ and $S_{1}, \ldots, S_{n}, S$ are sorts in $\Sigma$. When $n$ is $0, f$ is also called a constant symbol. We use the binary predicate $\approx$ to denote equality. We assume that $\Sigma$ always includes a Boolean sort Bool and constants true and false of that sort-allowing us to encode all other predicate symbols as function symbols of return sort Bool. For such symbols, we may write, e.g., $P\left(t_{1}, \ldots, t_{n}\right)$ as shorthand for the equality $P\left(t_{1}, \ldots, t_{n}\right) \approx$ true. A signature $\Sigma_{0}$ is a subsignature of a signature $\Sigma$, and $\Sigma$ is a supersignature of $\Sigma_{0}$, if every sort and function symbol of $\Sigma_{0}$ is also in $\Sigma$.

Given a signature $\Sigma$, a $\Sigma$-term is either a (sorted) variable $x$ with sort from $\Sigma$, or an expression of the form $f\left(t_{1}, \ldots, t_{n}\right)$, where $f$ is a function from $\Sigma$, and $t_{1}, \ldots, t_{n}$ are $\Sigma$-terms. A term $t$ is a well-sorted term of sort $S$ if $t$ is a variable having sort $S$, or $t$ is of the form $f\left(t_{1}, \ldots, t_{n}\right)$ where $f$ is of sort $S_{1} \times \cdots \times S_{n} \rightarrow S$, and $t_{1}, \ldots, t_{n}$ are well-sorted terms of sorts $S_{1}, \ldots, S_{n}$ respectively. An atomic $\Sigma$-formula is an equality $t_{1} \approx t_{2}$ where $t_{1}$ and $t_{2}$ are well-sorted $\Sigma$-terms of the same sort. A $\Sigma$-literal is either an atomic $\Sigma$-formula $p$ or its negation $\neg p$. We write $s \not t$ as an abbreviation for $\neg s \approx t$. A $\Sigma$-clause is a disjunction of $\Sigma$-literals, e.g. $l_{1} \vee \ldots \vee l_{n}$. A $\Sigma$-formula is an expression built from atomic $\Sigma$-formulas logical connectives such as $\vee, \wedge$, and $\neg$, and quantifiers $\forall$ and $\exists$. An occurrence of variable $x$ is free in a formula $\varphi$ if it does not reside within a subformula $\forall x \psi$ or $\exists x \psi$ of $\varphi$. We write $F V(\varphi)$ to denote the set of variables that occur free in $\varphi$, or the free variables of $\varphi$. A $\Sigma$-sentence is a $\Sigma$-formula with no free variables. A $\Sigma$ term or formula is ground if it contains no variables. More generally, by a slight abuse of terminology, we will sometimes call ground any quantifier-free term or formula. Where $\mathbf{x}=\left(x_{1}, \ldots, x_{n}\right)$ is a tuple of sorted variables, we write $\forall \mathbf{x} \varphi$ as an abbreviation for $\forall x_{1} \cdots \forall x_{n} \varphi$ and $\exists \mathbf{x} \varphi$ as an abbreviation for $\neg \forall \mathbf{x} \neg \varphi$. When using this notation, we will implicitly assume that $\mathbf{x}$ is maximal-for example we assume that $\forall x_{1} \forall x_{2} \varphi$ is instead written as $\forall x_{1} x_{2} \varphi$.

A substitution $\sigma$ is a mapping from variables to terms, applied in postfix form, such that $x \sigma$ and $x$ have the same sort for every variable $x$ and the set $\operatorname{Dom}(\sigma):=$ $\{x \mid x \sigma \neq x\}$, the domain of $\sigma$, is finite. We say $\sigma$ is a most general unifier (or $m g u$ ) of terms $t_{1}$ and $t_{2}$ if $\sigma$ is a substitution with minimal domain such that $t_{1} \sigma=t_{2} \sigma$.

A $\Sigma$-interpretation $\mathcal{I}$ maps each sort $S$ in $\Sigma$ to a non-empty set $S^{\mathcal{I}}$, the domain of $S$ in $\mathcal{I}$; it maps each variable $x$ of sort $S$ to an element $x^{\mathcal{I}}$ of $S^{\mathcal{I}}$ and each function symbol $f: S_{1} \times \cdots \times S_{n} \rightarrow S \in \Sigma$ to a total function $f^{\mathcal{I}}: S_{1}^{\mathcal{I}} \times \cdots \times S_{n}^{\mathcal{I}} \rightarrow S^{\mathcal{I}}$. If $\Sigma_{0}$ is a subsignature of $\Sigma$, the $\Sigma_{0}$-reduct of $\mathcal{I}$ is the $\Sigma_{0}$-interpretation $\mathcal{I}_{0}$ that interprets the symbols of $\Sigma_{0}$ exactly as $\mathcal{I}$ does. The evaluation of a term $f\left(t_{1}, \ldots, t_{n}\right)$ in $\mathcal{I}$, denoted $\mathcal{I} \llbracket t \rrbracket$, is defined recursively as $(i) \mathcal{I} \llbracket x \rrbracket=x^{\mathcal{I}} ;(i i) \mathcal{I} \llbracket f\left(t_{1}, \ldots, t_{n}\right) \rrbracket=$ $f^{\mathcal{I}}\left(\mathcal{I} \llbracket t_{1} \rrbracket, \ldots \mathcal{I} \llbracket t_{n} \rrbracket\right)$. For a $\Sigma$-interpretation $\mathcal{I}$, a variable $x$ of sort $S$, and an element $u$ of $S^{\mathcal{I}}$, we write $\mathcal{I}[x \rightarrow u]$ to denote a $\Sigma$-interpretation that interprets $x$ as $u$, and is otherwise identical to $\mathcal{I}$. The usual satisfiability relation $\models$ between $\Sigma$-interpretations and $\Sigma$-formulas, written $\models$, is defined as follow ${ }^{1}$

1 Cases for additional constructs such as $\Rightarrow, \Leftrightarrow$ and $\exists$ can be defined as usual by reduction to the cases below. 
- $\mathcal{I} \models t_{1} \approx t_{2}$ iff $\mathcal{I} \llbracket t_{1} \rrbracket=\mathcal{I} \llbracket t_{2} \rrbracket$

- $\mathcal{I} \models \varphi \wedge \psi$ iff $\mathcal{I} \models \varphi$ and $\mathcal{I} \models \psi$

- $\mathcal{I}=\varphi \vee \psi$ iff $\mathcal{I}=\varphi$ or $\mathcal{I}=\psi$

- $\mathcal{I} \models \neg \varphi$ iff $\mathcal{I} \not \models \varphi$

- $\mathcal{I} \models \forall x \varphi$ iff $\mathcal{I}[x \rightarrow v] \models \varphi$ for all $v \in S^{\mathcal{I}}$ where $S$ is the sort of $x$.

A $\Sigma$-interpretation $\mathcal{M}$ satisfies (or is a model of ) a $\Sigma$-formula $\varphi$ if $\mathcal{M}=\varphi ; \mathcal{M}$ satisfies (or is a model of) a set of $\Sigma$-formulas if it satisfies all of them. A $\Sigma$ formula or set of $\Sigma$-formulas is satisfiable if it has a model and is unsatisfiable otherwise. We consider only interpretations that interpret Bool as a binary set and true and false as distinct elements of that set. We write $\perp$ to abbreviate the unsatisfiable formula false $\approx$ true. A set $\Gamma$ of formulas propositionally entails a formula $\varphi$, written $\Gamma \models_{p} \varphi$, if the set $\Gamma \cup\{\neg \varphi\}$ is unsatisfiable when considering all atomic formulas in it as propositional (Boolean) variables.

A theory is a pair $T=(\Sigma, \mathbf{I})$ where $\Sigma$ is a signature and $\mathbf{I}$ is a class of $\Sigma$ interpretations, the models of $T$, closed under variable reassignment (that is, for all $\mathcal{I} \in \mathbf{I}$, every $\Sigma$-interpretation that differs from $\mathcal{I}$ only in how it interprets variables is also in $\mathbf{I})$. The union of two theories $T_{1}=\left(\Sigma_{1}, \mathbf{I}_{1}\right)$ and $T_{2}=\left(\Sigma_{2}, \mathbf{I}_{2}\right)$, when it exists, is the theory $T_{1} \cup T_{2}=(\Sigma, \mathbf{I})$ where $\Sigma$ is the smallest supersignature of $\Sigma_{1}$ and $\Sigma_{2}$ and $\mathbf{I}$ is the set of all $\Sigma$-interpretations whose $\Sigma_{i}$-reduct is in $\mathbf{I}_{i}$ for $i=1,2$. This definition extends to more than two theories as expected.

Given a theory $T=(\Sigma, \mathbf{I})$, a $\Sigma$-formula $\varphi$ is satisfiable modulo $T$, or $T$ satisfiable, if and only if there is a model of $T$ that satisfies $\varphi$. A set $\Gamma$ of $\Sigma$-formulas $T$-entails a $\Sigma$-formula $\varphi$, written $\Gamma \models_{T} \varphi$, if every model of $T$ that satisfies all formulas in $\Gamma$ satisfies $\varphi$ as well. The formula $\varphi$ is $T$-valid if it is $T$-entailed by the empty set - equivalently, if it is satisfied by every model of $T$. Two sets $\Gamma_{1}$ and $\Gamma_{2}$ of $\Sigma$-formulas are equisatisfiable in $T$ if for every model of $T$ that satisfies one there is a model of $T$ that satisfies the other, and the two models differ at most on the way they interpret the free variables not shared by $\Gamma_{1}$ and $\Gamma_{2}$.

\section{The $\operatorname{DPLL}\left(T_{1}, \ldots, T_{m}\right)$ Framework}

Most SMT solvers have a basic architecture that combines in a principled way a propositional satisfiability solver, the SAT engine, with a number of theory solvers, specialized constraint solvers for sets of literals over a specific theory. A general framework, called DPLL $(T)$, to describe at an abstract but formal level the working of such SMT solvers and the interaction of their main components was originally developed by Nieuwenhuis et al. 26. The framework, parametrized by a background theory $T$, describes entire families of procedures to determine the $T$ satisfiability of a ground set of input clauses. We present here a variant of it, introduced by Krstić and Goel [23], where $T$ is not a monolithic theory but is instead the union of a number of separate sub-theories, each with its own theory solver.

\subsection{The theory $T$}

For the rest of the paper, we will consider a $\Sigma$-theory $T=T_{1} \cup \cdots \cup T_{m}$ where each $T_{i}$ is a theory with signature $\Sigma_{i}$. One of these theories, say $T_{e}$ with $e \in\{1, \ldots, m\}$, 
may be the theory of equality - over the symbols $\Sigma_{e}$. This theory, whose set of models consists of all $\Sigma_{e}$-interpretations, is also known as the theory of equality with uninterpreted functions (EUF). As a consequence, we will refer to the sort and function symbols of $T$ that occur only in $\Sigma_{e}$ as uninterpreted and to the other symbols of $T$ as interpreted. To stress that we treat the component theories of $T$ individually we will refer to our variant of $\operatorname{DPLL}(T)$ as $\operatorname{DPLL}\left(T_{1}, \ldots, T_{m}\right)$.

For convenience and without loss of generality, we assume that if a signature from $\left\{\Sigma_{1}, \ldots, \Sigma_{m}\right\}$ shares a sort symbol with another signature then it shares it also with all the signatures in the set. Finally, we impose the (true) restriction that the signatures $\Sigma_{1}, \ldots, \Sigma_{m}$ share no function symbols at all except for true and false. This restriction is currently imposed by all SMT solvers that support multiple theories as it enables the modular combination of theory solvers for the individual theories.

\subsection{Transition system}

The $\operatorname{DPLL}\left(T_{1}, \ldots, T_{m}\right)$ framework for theory $T$ defines a state transition system for each ground $\Sigma$-formula $\varphi_{0}$ whose $T$-satisfiability one is interested in. Intuitively, the initial state of the system corresponds to a CNF encoding of $\varphi_{0}$. Under the right conditions on $T$, all of the executions of the system starting from such a state end in a distinguished fail state if and only if $\varphi_{0}$ is not $T$-satisfiable.

States System states are all triples of the form $\langle M, F, C\rangle$ where

- $M$, the current assignment, is a sequence of literals and decision points $\bullet$,

$-F$ is a set of ground clauses derived from $\varphi_{0}$, and

$-C$ is either the distinguished value no or a clause, which we will refer to as a conflict clause.

Each assignment $M$ can be factored uniquely into the subsequence concatenation $M_{0} \bullet M_{1} \bullet \cdots \bullet M_{n}$, where no $M_{i}$ contains decision points. For $i=0, \ldots, n$, we call $M_{i}$ the decision level $i$ of $M$ and denote with $M^{[i]}$ the subsequence $M_{0} \bullet \cdots \bullet M_{i}$. When convenient, we will treat $M$ as the set of its literals. The formulas in $F$ have a particular purified form that can be assumed with no loss of generality since any formula can be efficiently converted into that form while preserving equisatisfiability in $T$ : each element of $F$ is a ground clause, and each atom occurring in $F$ is pure, that is, has signature $\Sigma_{i}$ for some $i \in\{1, \ldots, m\}$. By the way assignments are constructed, their atoms too are always pure.

Initial states have the form $\left\langle\emptyset, F_{0}\right.$, no $\rangle$ where $F_{0}$ is an input set of clauses to be checked for $T$-satisfiability. The expected final states are states of the form $\langle M, F, \perp\rangle$, when $F_{0}$ is not $T$-satisfiable; or else $\langle M, F$, no $\rangle$ with $M$ satisfiable in $T$, $F$ equisatisfiable with $F_{0}$ in $T$, and $M={ }_{\mathrm{p}} F$.

If $M$ is $T$-satisfiable and $M \models_{\mathrm{p}} F$ we call $M$ a satisfying assignment for $F$.

Transition rules The possible behaviors of the system are defined by a set of nondeterministic state transition rules, specifying a set of successor states for each current state. The rules are provided in Figure 1 in guarded assignment form [23]. A rule applies to a state $s$ if all of its premises hold for $s$. In the rules, $M, F$ and $C$ respectively denote the assignment, formula set, and conflict clause component 
Propagate $_{i} \frac{l_{1}, \ldots, l_{n} \in M \quad l_{1}, \ldots, l_{n} \models_{i} l \quad l \in \operatorname{Lit}_{F} \cup \operatorname{Int}_{M} \quad l, \bar{l} \notin M}{M:=M l}$

Decide $\frac{l \in \operatorname{Lit}_{F} \cup \operatorname{Int}_{M} \quad l, \bar{l} \notin M}{M:=M \bullet l} \quad$ Conflict $_{i} \frac{C=\text { no } \quad l_{1}, \ldots, l_{n} \in M \quad l_{1}, \ldots, l_{n} \models{ }_{i} \perp}{C:=\bar{l}_{1} \vee \cdots \vee \bar{l}_{n}}$

$\operatorname{Explain}_{i} \frac{C=l \vee D \quad \bar{l}_{1}, \ldots, \bar{l}_{n} \models_{i} \bar{l} \quad \bar{l}_{1}, \ldots, \bar{l}_{n} \prec_{M} \bar{l}}{C:=l_{1} \vee \cdots \vee l_{n} \vee D}$

$\operatorname{Learn}_{i} \quad \frac{\emptyset \models_{i} l_{1} \vee \cdots \vee l_{n} \quad l_{1}, \ldots,\left.l_{n} \in \operatorname{Lit}_{M}\right|_{i} \cup \operatorname{Int}_{M} \cup L_{i}}{F:=F \cup\left\{l_{1} \vee \cdots \vee l_{n}\right\}} \quad$ Learn $_{0} \quad \frac{C \neq \text { no } \bullet \in M}{F:=F \cup\{C\}}$

Backjump $\frac{C=l_{1} \vee \cdots \vee l_{n} \vee l \quad \text { lev } \bar{l}_{1}, \ldots, \text { lev } \bar{l}_{n} \leq i<\operatorname{lev} \bar{l}}{C:=\text { no } M:=M^{[i]} l} \quad$ Fail $\quad \frac{C \neq \text { no } \bullet \notin M}{C:=\perp}$

Fig. $1 \operatorname{DPLL}\left(T_{1}, \ldots, T_{m}\right)$ rules

of the current state. The conclusion describes how each component is changed, if at all.

We write $\bar{l}$ to denote the complement of literal $l$ and write $l \prec_{M} l^{\prime}$ to indicate that $l$ occurs before $l^{\prime}$ in $M$. The function lev maps each literal of $M$ to the (unique) decision level at which $l$ occurs in $M$. The set $\operatorname{Lit}_{F}$ (resp., $\operatorname{Lit}_{M}$ ) consists of all literals in $F$ (resp., all literals in $M$ ) and their complements. For $i=1, \ldots, m$, the set $\left.\operatorname{Lit}_{M}\right|_{i}$ consists of the $\Sigma_{i}$-literals of $\operatorname{Lit}_{M}$. Int $M$ is the set of all interface literals of $M$ : the equalities and disequalities between shared variables where the set of shared variables is

$\left\{x \mid x\right.$ is a variable in both $\left.\operatorname{Lit}_{M}\right|_{i}$ and $\left.\operatorname{Lit}_{M}\right|_{j}$ for some $\left.1 \leq i<j \leq m\right\}$.

The index $i$ in the rules ranges from 0 to $m$ for Propagate $_{i}$, Conflict $_{i}$ and

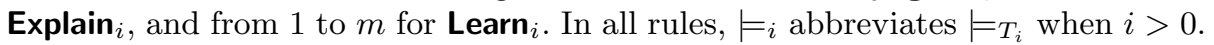
In Propagate Pr $_{1}, \ldots, l_{n} \models_{0} l$ simply means that $\bar{l}_{1} \vee \cdots \vee \bar{l}_{n} \vee l \in F$. Similarly, in Conflict $_{0}, l_{1}, \ldots, l_{n} \models_{0} \perp$ means that $\bar{l}_{1} \vee \cdots \vee \bar{l}_{n} \in F$; in $\operatorname{Explain}_{0}, \bar{l}_{1}, \ldots, \bar{l}_{n} \models{ }_{0} \bar{l}$ means that $l_{1} \vee \cdots \vee l_{n} \vee \bar{l} \in F$.

The rules Decide, Propagate , Explain $_{0}$, Conflict $_{0}$, Learn, and Backjump model the behavior of the SAT engine, which treats atoms as Boolean variables. The rules Conflict $_{0}$ and Explain $_{0}$ model the conflict discovery and analysis mechanism used by CDCL SAT solvers. All the other rules model the interaction between the SAT engine and the individual theory solvers in the overall SMT solver.

Generally speaking, the system uses the SAT engine to construct the assignment $M$ as if the problem were propositional, but it periodically asks the subsolvers for each theory $T_{i}$ to check if the set of $\Sigma_{i}$-literals in $M$ is $T_{i}$-unsatisfiable, or entails in $T_{i}$ some yet undetermined literal from $\operatorname{Lit}_{F} \cup \operatorname{Int}_{M}$. In the first case, the sub-solver returns an explanation of the unsatisfiability as a conflict clause, which is modeled by Conflict C $_{i}$ with $i \in\{1, \ldots, m\}$. The propagation of entailed theory literals and the extension of the conflict analysis mechanism to them is modeled by the rules Propagate $_{i}$ and Explain $_{i}$.

The inclusion of the interface literals $\operatorname{Int}_{M}$ in Decide and Propagate Pachieves the effect of the Nelson-Oppen combination method [9,35. Under the right conditions on the component theories, the two rules allows the overall system to determine the $T$-satisfiability of the input formula by doing only local reasoning 
in the individual component theories and exchanging information between their corresponding solvers just through (dis)equalities between interface variables.

The rule Learn $\mathbf{n}_{i}$ with $i>0$ is needed to model theory solvers following the splitting-on-demand paradigm [3. When asked about the satisfiability of their constraints, these solvers may instead return a splitting lemma, a $T_{i}$-valid formula that encodes an additional guess that needs to be made about the literals in $M$ before the solver can determine their satisfiability. The set $L_{i}$ in the rule is a finite set consisting of literals, not present in the input set $F_{0}$, which may be generated by such solvers.

\subsection{System executions and correctness}

An execution of a transition system modeled as above is a (possibly infinite) sequence $s_{0}, s_{1}, \ldots$ of states such that $s_{0}$ is an initial state and for all $i \geq 0, s_{i+1}$ can be generated from $s_{i}$ by the application of one of the transition rules. A system state is reachable if it occurs in some execution; it is irreducible if no transition

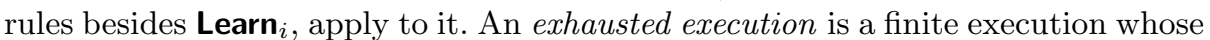
last state is irreducible. A complete execution is either an exhausted execution or an infinite execution. An application of Learn $_{i}$, with $i \geq 0$, is redundant in an execution if the execution contains a previous application of Learn $_{i}$ with the same premise.

Adapting results from [3,23,26], it can be shown that every execution starting with a state $\left\langle\emptyset, F_{0}\right.$, no $\rangle$ and ending in $\langle M, F, C\rangle$ satisfies the following invariants:

1. $M$ contains only pure literals and no repetitions;

2. $F \models_{T} C$ and $M \models_{\mathrm{p}} \neg C$ when $C \neq$ no;

3. $F_{0}$ and $F$ are equisatisfiable in $T$.

Moreover, the transition system is terminating: every execution with no redundant

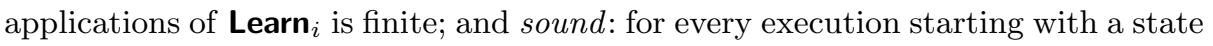
$\left\langle\emptyset, F_{0}\right.$, no $\rangle$ and ending with a state $\langle M, F, \perp\rangle$, the clause set $F_{0}$ is $T$-unsatisfiable. Under suitable assumptions on the sub-theories $T_{1}, \ldots, T_{m}$, the system is also complete: for every exhausted execution starting with $\left\langle\emptyset, F_{0}\right.$, no $\rangle$ and ending with $\langle M, F$, no $\rangle, M$ is satisfiable in $T$ and $M \models_{\mathrm{p}} F_{0}$. Here, we provide a sketch of the correctness proof for $\operatorname{DPLL}\left(T_{1}, \ldots, T_{m}\right)$ restricted to a single theory $T_{1}$ based on the proof for the original framework [26].

Theorem 1 Suppose $T=T_{1}$. With any strategy where all applications of Learn $\mathbf{L}_{1}$ are not redundant and introduce new literals only from a finite set $L_{1}, \operatorname{DPLL}\left(T_{1}\right)$ is sound, complete, and terminating for all sets $F_{0}$ of ground clauses.

\section{Proof: (Sketch)}

Soundness) Observe that all reachable states of the form $\langle M, F, C\rangle$ where $C \neq$ no are such that $C$ is $T_{1}$-entailed by $F_{0}$. When $C$ is introduced by Conflict $_{0}$, it is a clause from $F$; when it is introduced by Conflict $_{1}$, it is $T_{1}$-valid. When applying $\operatorname{Explain}_{i}$, we replace a literal $l$ in $C$ with disjunction of literals $l_{1} \vee \cdots \vee l_{n}$ which is entailed by $l$ either in the theory $T_{1}$ (when $i=1$ ), or together with $F$ (when $i=0)$. Thus, when a state of the form $\langle M, F, \perp\rangle$ is reachable, we can conclude that $F \mid=_{T_{1}} \perp$. Since all clauses in $F$ are $T_{1}$-entailed by $F_{0}$ by construction, $\perp$ is $T_{1}$-entailed by $F_{0}$ as well. 


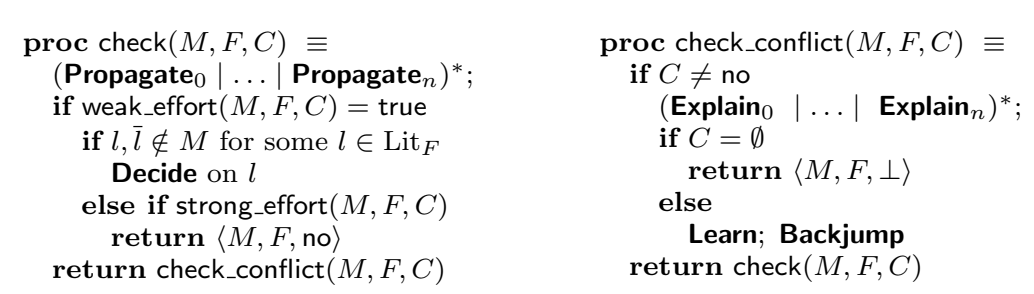

Fig. 2 A typical strategy check for applying $\operatorname{DPLL}\left(T_{1}, \ldots, T_{m}\right)$ rules.

Termination) For all reachable states $\langle M, F, C\rangle$, every literal occurring in $M$, $F$ or $C$ belongs to the finite set of literals $\operatorname{Lit}_{M} \cup \operatorname{Int}_{M} \cup L_{1}$. As a consequence, there is only a finite number of reachable states. Consider a partial ordering $\succeq$ on assignments $M$, with the empty assignment as maximal element, such that $\left(e_{1} M_{1}\right) \succeq\left(e_{2} M_{2}\right)$ if either $(i) e_{1}=\bullet$ and $e_{2} \neq \bullet$ or $(i i) e_{1}=e_{2}$ and $M_{1} \succeq M_{2}$. In addition, consider a partial ordering $\succeq$ on conflict clauses, seeing as sets of literals, such that $C_{1} \succeq C_{2}$ if either $C_{1}$ is no, or neither $C_{1}$ nor $C_{2}$ are no and $C_{2} \prec_{M}^{m u l} C_{1}$, where $\prec_{M}^{m u l}$ is the multiset extension of $\prec_{M}$. Extend this ordering to states so that $\left\langle M_{1}, F_{1}, C_{1}\right\rangle \succeq\left\langle M_{2}, F_{2}, C_{2}\right\rangle$ if and only if $M_{1} \succeq M_{2}$, or $M_{1}=M_{2}$ and $C_{1} \succeq C_{2}$. One can show that this ordering is well founded. Moreover, applying all rules besides Learn L $_{1}$ to a state $s$ results in a state $s^{\prime}$ where $s \succ s^{\prime}$. Termination

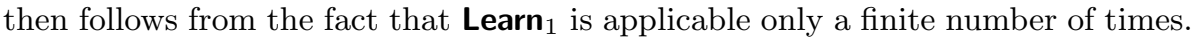

Completeness) We claim that for every irreducible reachable state $\langle M, F$, no $\rangle$, $M$ is a $T_{1}$-satisfiable satisfying assignment for $F$. To see this, consider first that since Decide does not apply to the state, $M$ must contain an assignment for all literals in $F$. Moreover, $M$ is a satisfying assignment for $F$ since Conflict . $_{0}$ does not apply. Since Conflict C $_{1}$ does not apply, then $M$ must be $T_{1}$-satisfiable. Since $F_{0} \subseteq F$, we have that $M$ propositionally entails $F_{0}$, and thus $F_{0}$ is $T_{1}$-satisfiable as well. Thus, since our procedure is terminating, it is also complete.

The soundness and termination arguments in the proof above immediately extend to the multiple theory case of $\operatorname{DPLL}\left(T_{1}, \ldots, T_{m}\right)$ where $m>1$. The completeness argument can be extended as well under further model-theoretic assumptions on the component theories [23,20].

\subsection{A Typical Strategy for $\operatorname{DPLL}\left(T_{1}, \ldots, T_{m}\right)$}

A typical strategy for applying the theory-specific rules of $\operatorname{DPLL}\left(T_{1}, \ldots, T_{m}\right)$ is outlined in Figure 2 where $\mid$ denotes alternative choice and ${ }^{*}$ denotes zero or more rule applications. The check procedure involves two sub-procedures weak_effort and strong_effort, which are not shown here and are specific to the theory $T$. Each of these methods when invoked either applies Conflict Cor $_{i}$ Learn ${ }_{i}$ for some $1 \leq i \leq m$ and returns false, or applies no rules and returns true.

Weak effort checks, as denoted by weak_effort, are commonly used to eagerly avoid extensions that are clearly unsatisfiable in one of the theories. On the other hand, strong effort checks, as denoted by strong_effort, are required to make progress towards determining the $T$-satisfiability of the conjunction of literals in $M$. In particular, unlike weak_effort, we require that strong_effort returns true 
only when $M$ is $T$-satisfiable. Generally speaking, weak effort checks typically involve computationally inexpensive reasoning at the cost of incompleteness, whereas strong effort checks are complete but may involve expensive reasoning. The design of a theory solver in the $\operatorname{DPLL}\left(T_{1}, \ldots, T_{m}\right)$ framework depends largely on how the methods weak_effort and strong_effort are implemented. We will see an example of these functions in Section 6.2.

In more detail, the first sub-procedure check in Figure 2 applies to states $\langle M, F, C\rangle$ where $C=$ no. We first apply the rule Propagate Pr $_{i}$ for sub-theories $T_{i}$, possibly multiple times. Afterwards, we apply a weak effort check. If no conflicts or clauses are learned at weak effort, we apply Decide on some unassigned literal $l$ from $\operatorname{Lit}_{F}$, if one exists. Otherwise, our assignment $M$ is complete, and we apply a strong effort check to verify the $T$-satisfiability of $M$. If strong_effort $(M, F, C)$ returns true, then $M$ is satisfiable in $T$, and the method returns the (final) state $\langle M, F$, no $\rangle$, indicating that $F$ is satisfiable. In all other cases, we apply check_conflict.

The second sub-procedure check_conflict is applied to states $\langle M, F, C\rangle$ where $C$ may be different from no. In those cases, we perform conflict analysis by repeated applications of $\operatorname{Explain}_{i}$. If we reach the fail state, then we know $F$ is unsatisfiable. Otherwise, we add a learned clause via Learn, and apply Backjump to return to a prefix of $M$.

We formally state the requirements of weak and strong effort checks for the single theory case in the following proposition which is a consequence of Theorem 1 .

Proposition 1 Suppose $T=T_{1}$. The check method in Figure 2 implements a sound, complete, and terminating strategy for all sets $F_{0}$ of ground clauses provided all of the following hold.

1. In weak_effort and strong_effort, all applications of Learn L $_{1}$ are not redundant and introduce new literals only from a finite set $L_{1}$.

2. weak_effort and strong_effort return false only when they apply at least one rule.

3. strong_effort $(M, F, C)$ returns true only when the conjunction of the literals in $M$ is $T_{1}$-satisfiable.

Proof: (Sketch) The first point ensures that check meets the requirements on applications of Learn 1 as given in Theorem 1 the second point ensures that check is a terminating method; and the third point ensures that check generates exhaustive executions.

\section{Finite Model Finding in SMT}

The $\operatorname{DPLL}\left(T_{1}, \ldots, T_{m}\right)$ framework described in the previous section is limited to quantifier-free formulas. This section outlines an approach for finite model finding for quantified formulas that can be integrated in $\operatorname{DPLL}\left(T_{1}, \ldots, T_{m}\right)$-based SMT solvers. Concretely, we consider $\Sigma$-formulas in the following language:

$$
\phi:=t_{1} \approx t_{2}\left|\phi_{1} \wedge \phi_{2}\right| \phi_{1} \vee \phi_{2}|\neg \phi| \forall x \varphi
$$

where $t_{1}$ and $t_{2}$ are $\Sigma$-terms, and the sort of $x$ is either an uninterpreted sort or a sort interpreted in every model of $T$ as a finite set of some fixed cardinality. Examples of the latter include sorts denoting fixed-length bit-vectors or finite (non-recursive) datatypes. Certain integer arithmetic constraints with bounded 
quantifiers, where the bounds are explicitly provided or can be inferred, can be treated similarly to finite interpreted sorts [32,5]. Many applications of SMT rely on solving problems that fall into such categories, given a careful encoding of the constraints.

Given an input formula $\psi$ in the grammar above, our approach first performs a purification step, which results in a set $F$ of ground clauses, and a set $A$ of equivalences of the form $a \approx$ true $\Leftrightarrow \forall \mathbf{x} \varphi$, abbreviated as $a \Leftrightarrow \forall \mathbf{x} \varphi$, where $a$ is a Boolean variable uniquely associated with the quantified formula $\forall \mathbf{x} \varphi$. We will refer to $a$ as the proxy variable for $\forall \mathbf{x} \varphi$. The set $F$ can be constructed by a standard conversion of $\psi$ to clausal form which, however, treats the quantified subformulas of $\psi$ as atoms. After that, each quantified formula $\forall \mathbf{x} \varphi$ occurring in a clause of $F$ is replaced with its proxy variable $a$ if it occurs positively in the clause, and with $\varphi\{\mathbf{x} \mapsto \mathbf{k}\}$ otherwise, where $\varphi\{\mathbf{x} \mapsto \mathbf{k}\}$ is the result of substituting each occurrence in $\varphi$ of a variable $x$ of $\mathbf{x}$ with a fresh variable $k$ (to be treated like a Skolem constant). The process is repeated until $F$ contains no quantifiers. This conversion from $\psi$ to $F$ and $A$ can be done so that $\psi$ and $F \cup A$ are equisatisfiable. We will denote by $\lfloor\psi\rfloor$ the resulting pair $(F, A)$.

Example 1 Consider the formula $\psi=\neg P(b, c) \wedge(Q(b, c) \Leftrightarrow \forall x P(b, x))$ where $P$ and $Q$ are uninterpreted predicates and $x, b$ and $c$ are of some uninterpreted sort $S$. The purified form $\lfloor\psi\rfloor$ is computed as follows. First, a conversion of $\psi$ to clausal normal form results in the clauses:

$$
F_{0}:=\{\neg P(b, c), \neg Q(b, c) \vee \forall x P(b, x), Q(b, c) \vee \neg \forall x P(b, x)\}
$$

We replace the occurrence of $\neg \forall x P(b, x)$ in the third clause of $F_{0}$ with $\neg P(b, k)$, where $k$ is a fresh variable of sort $S$, and replace the positive occurrence in the second clause with a fresh proxy variable $a$ of sort Bool. We obtain the quantifierfree set of purified clauses $F$ and equivalences $A$ :

$$
\begin{aligned}
F & :=\{\neg P(b, c), \neg P(b, k) \vee Q(b, c), a \vee \neg Q(b, c)\} \\
A & :=\{a \Leftrightarrow \forall x P(b, x)\}
\end{aligned}
$$

It is not hard to see that $\phi$ and $F \cup A$ are equisatisfiable in $T$.

\section{Model finding procedure}

Figure 3 describes a finite model finding procedure called FM-Solve $e_{\mathcal{H}}$ that takes as input a set $F$ and a set $A$, where $(F, A)=\lfloor\phi\rfloor$ for some $\Sigma$-formula $\psi$, and tries to determine the satisfiability of $F \cup A$ by adding to $F$ instances of the quantified formulas that occur in $A$. The procedure is parametrized by an instantiation heuristic $\mathcal{H}$ for the quantified formulas.

In Step 1, it looks for a satisfying assignment $M$ for $F 2^{2}$ This assignment can be found using the $\operatorname{DPLL}\left(T_{1}, \ldots, T_{m}\right)$ procedure from the previous section. If no satisfying assignment can be found, the procedure terminates with unsat, for "unsatisfiable." Otherwise, in Step 2, it constructs a $\Sigma$-interpretation $\mathcal{M}$ that

\footnotetext{
2 Recall that a satisfying assignment is a $T$-satisfiable set of $\Sigma$-literals that propositionally entail $F$.
} 
FM-Solve $_{\mathcal{H}}(F, A)$

Input: A set $F$ of purified $\Sigma$-clauses and a set $A$ of equivalences

Output: sat or unsat

1. Find a satisfying assignment $M$ for $F$. If none is found, return unsat.

2. Construct a $\Sigma$-interpretation $\mathcal{M}$ that satisfies $M$. Let $\mathbf{V}$ be a minimal set of $\Sigma$-terms such that, for each uninterpreted sort $S$ of $\Sigma$, every element of $S^{\mathcal{M}}$ is denoted by a term in $\mathbf{V}$ (that is, for all $s \in S^{\mathcal{M}}$ there is a $v \in \mathbf{V}$ such that $s=\mathcal{M} \llbracket v \rrbracket$ ).

3. For each $\forall \mathbf{x} \varphi$ where $a \Leftrightarrow \forall \mathbf{x} \varphi \in A$ and $a \in M$,

(a) let $I_{\mathbf{x}}$ be the set of substitutions from $\mathbf{x}$ to terms in $\mathbf{V}$ chosen by $\mathcal{H}(\mathcal{M}, \forall \mathbf{x} \varphi)$;

(b) let $(F, A)=\left(F \cup F^{\prime}, A \cup A^{\prime}\right)$ where $\left(F^{\prime}, A^{\prime}\right)=\left\lfloor\left\{\neg a \vee \varphi \sigma \mid \sigma \in I_{\mathbf{x}}\right\}\right\rfloor$.

If each of the sets $I_{\mathbf{x}}$ was empty, return sat, otherwise go to Step 1.

Fig. 3 Finite Model Finding Procedure FM-Solve $\mathcal{H}$, parameterized by a quantifier instantiation heuristic $\mathcal{H}$.

satisfies $M$. In doing so, however, it considers only $\Sigma$-interpretations $\mathcal{M}$ that interpret the uninterpreted sorts of $\Sigma$ as finite sets. This makes it feasible to actually construct the set $\mathbf{V}$ used in Step 3. In that step, the procedure considers the set of quantified formulas that are active in $M$, that is, those whose proxy variable occurs positively in $M$. It adds new constraints $F^{\prime} \cup A^{\prime}$ to $F \cup A$ based on instances of quantified formulas chosen by the heuristic $\mathcal{H}$, which takes as input a model and a quantified formula. We consider only heuristics that are sound with respect to models: if $\mathcal{H}$ returns no instances for quantified formulas in Step 3, it is because $\mathcal{M}$ satisfies all active quantified formulas in $M$, and so the procedure terminates with sat, for "satisfiable."

Theorem 2 For all inputs $F, A$ for $\mathrm{FM}^{-S_{0}} \mathrm{lve}_{\mathcal{H}}$, the following hold.

1. If the method for finding satisfying assignments $M$ for $F$ in Step 1 is sound, then the procedure $\mathrm{FM}-S_{\mathrm{S}} \mathrm{ve}_{\mathcal{H}}$ returns unsat only if $F \cup A$ is $T$-unsatisfiable.

2. If for all inputs, $\mathcal{H}(\mathcal{M}, \forall \mathbf{x} \varphi)$ returns the empty set only if $\mathcal{M} \models \forall \mathbf{x} \varphi$, then the procedure $\mathrm{FM}-\mathrm{Solve}_{\mathcal{H}}$ returns sat only if $F \cup A$ is $T$-satisfiable.

Proof: To show Point 1, assume the method for finding satisfying assignments $M$ for $F$ in Step 1 is sound. Thus, when the procedure returns unsat, we have that $F$ is $T$-unsatisfiable. Since the formulas added to $F$ and $A$ in Step 3 preserve satisfiability, we have that our input is $T$-unsatisfiable as well.

To show Point 2, the procedure returns sat when $\mathcal{H}(\mathcal{M}, \forall \mathbf{x} \varphi)$ returns the empty set for all quantified formulas where $a \Leftrightarrow \forall \mathbf{x} \varphi \in A$ and $a \in M$. Assume $\mathcal{M}=\forall \mathbf{x} \varphi$ for all such formulas. Then, $F \cup A$ is satisfied by a model $\mathcal{M}^{\prime}$ where $a^{\mathcal{M}^{\prime}}=(\forall \mathbf{x} \varphi)^{\mathcal{M}}$ for each $a \Leftrightarrow \forall \mathbf{x} \varphi \in A$ and $a \notin M$, and where all other symbols are interpreted as in $\mathcal{M}$. Since during all iterations of the procedure $F \cup A$ remains a superset of the original input, we have that the latter is satisfied by $\mathcal{M}^{\prime}$ as well.

The following sections will examine in more detail the main ideas behind the three steps of procedure FM-Solve $\mathcal{H}_{\mathcal{H}}$. In Section 6, we describe techniques for finding satisfying assignments in Step 1. These assignments have models that interpret 
uninterpreted sorts as sets of minimal cardinality and are used in Step 2. In Section 7. we describe methods for constructing such models. Finally, in Section 8 , we describe quantifier instantiation heuristics that can be used to choose sets of substitutions for Step 3.

Although Theorem 2 holds in general for inputs that involve several theories, for simplicity, we restrict ourselves in the following to problems in EUF only, that is, involving only uninterpreted sorts and function symbols. Under these restrictions, we provide arguments for the correctness of the three steps of FM-Solve $\mathcal{H}$ in Theorems 4 and 5, which ensure the correctness of our finite model finding procedure according to Theorem 2 .

\section{EUF with Finite Cardinality Constraints (FCC)}

In this section, we introduce techniques for finding satisfying assignments in Step 1 of procedure $\mathrm{FM}-\mathrm{Solve}_{\mathcal{H}}$ from Figure 3 . We will focus on satisfying assignments that have small models, that is, models which interpret the uninterpreted sorts of our signature as finite sets of minimal size. To do this, we introduce an extension of the theory EUF with finite cardinality constraints (FCC). We describe its signature $\left(\Sigma_{\mathrm{FCC}}\right)$ and semantics, give a satisfiabiliy procedure for conjunctions of literals in this theory, and describe how it can be integrated into the $\operatorname{DPLL}\left(T_{1}, \ldots, T_{m}\right)$ architecture. Finally, we discuss a strategy, fixed-cardinality check $_{\mathrm{FCC}}$, which ensures that upper bounds are incrementally established for all uninterpreted sorts.

Definition 1 (FCC) Let EUF be the theory of equality and uninterpreted functions over some signature $\Sigma_{\mathrm{EUF}}$. The theory FCC of EUF with finite cardinality constraints is the extension of EUF obtained as follows. The signature $\Sigma_{\mathrm{FCC}}$ of FCC extends $\Sigma_{\text {EUF }}$ with a constant card ${ }_{S, k}$ of sort Bool for each sort $S$ of $\Sigma_{\text {EUF }}$ and integer $k>0$. Its models are all $\Sigma_{\mathrm{FCC}}$-interpretations that satisfy each atomic formula $\operatorname{card}_{S, k}$ exactly when they interpret $S$ as a set of cardinality at most $k$.

As shown below, the FCC-satisfiability of sets of $\Sigma_{\mathrm{FCC}}$-literals is a decidable problem. By a reduction from graph (vertex) coloring, one can show that the problem is NP-hard. The main idea of the reduction is to represent the set of $k$ colors as a sort $\mathrm{C}$ and represent the vertices of the graph as variables of sort $\mathrm{C}$. An edge between two vertices $x$ and $y$ is encoded as the constraint $x \not y$. The cardinality constraint on $\mathrm{C}$ is encoded by $\operatorname{card}_{\mathrm{C}, k}$. It is not difficult to see that given a model $\mathcal{M}$ of FCC (which is finitely representable), checking whether $\mathcal{M}$ satisfies a set of $\Sigma_{\mathrm{FCC}}$-literals can be done in polynomial time. It follows that this satisfiability problem is NP-complete.

We prove its decidability by providing an effective satisfiability procedure. The procedure relies on computing certain congruence closures of sets of constraints, so we start by introducing that notion.

Definition 2 (Congruence Closure) Let $M$ be a set of literals, in any signature, and let $\mathbf{T}_{M}$ be the set of all terms (and subterms) occurring in $M$. The congruence closure $M^{*}$ of $M$ is the smallest set of literals such that

1. $M \subseteq M^{*} \subseteq\left\{s \approx t \mid s, t \in \mathbf{T}_{M}\right\} \cup\left\{s \not t t \mid s, t \in \mathbf{T}_{M}\right\}$;

2. for all $s, t \in \mathbf{T}_{M}, M^{*} \models$ EUF $s \approx t$ iff $s \approx t \in M^{*}$. 


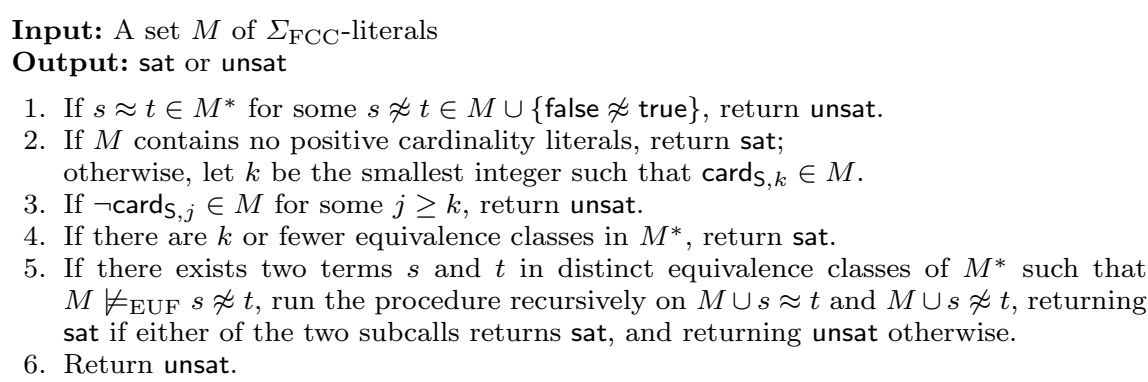

Fig. 4 Decision Procedure for FCC.

By construction, the relation $\left\{(s, t) \mid s \approx t \in M^{*}\right\}$ induced by $M^{*}$ is a congruence, and hence an equivalence, relation over $\mathbf{T}_{M}$. For brevity, we will identify $M^{*}$ with its induced equivalence relation when convenient. It can be shown (see, e.g., [1) that $(i) M^{*}$ is computable whenever $M$ is finite, and (ii) if $M$ is satisfiable it is satisfied by an interpretation $\mathcal{M}$ that interprets each sort $S$ as the set $\mathbf{V}_{S}=\left\{v_{1}^{S}, \ldots, v_{n_{S}}^{S}\right\}$ consisting of an arbitrary representative $v_{i}^{S}$ for each of the $n_{S}$ equivalence classes of $M^{*}$ over terms of sort $S$. We call $\mathcal{M}$ a normal model of $M$. Our procedure will seek to find normal models for given input sets $M$ of literals.

\subsection{Decision Procedure}

This section presents a decision procedure for the satisfiability of sets of constraints in the theory FCC. For now, we limit ourselves to signatures $\Sigma_{\text {FCC }}$ whose set of sorts consists of a single (uninterpreted) sort S. Figure 4 gives the decision procedure for the satisfiability problem in this case. As input, the procedure takes a set $M$ consisting of cardinality constraint literals for $\mathrm{S}$ and equalities and disequalities over ground $\Sigma_{\mathrm{FCC}}$-terms of sort $\mathrm{S}$, and terminates with sat or unsat.

Lemma 1 The procedure in Figure 4 is sound, complete and terminating for every set $M$ of $\Sigma_{\mathrm{FCC}}$-literals.

Proof: Soundness) Let us start by observing that splitting the problem based on equalities $s \approx t$, as done in Step 5 of the procedure, is trivially sound since all models of FCC satisfy exactly one of $s \approx t$ and $s \neq t$. The procedure answers unsat in one of the following cases:

1. an equality $s \approx t$ is entailed by $M$ where $s \not t t$ is also in $M \cup\{$ false $\not \approx$ true $\}$,

2. conflicting literals card $_{\mathrm{S}, k}$ and $\neg \operatorname{card}_{\mathrm{S}, j}$ are asserted for $j \geq k$, or

3 . there exist $k+1$ terms (each in a different equivalence class) that are entailed to be mutually disequal by $M$.

For the first case, it is immediate that $M$ has no models. For conflicts in the second case, no model can be constructed with both at most $k$ and at least $j+1$ elements in the domain of $\mathrm{S}$. For conflicts in the third case, note that if the procedure reaches Step 6 there must be $k+1$ equivalence classes with representatives $t_{1}, \ldots, t_{k+1}$, 
say, where $M \models$ EUF $t_{i} \not t_{j}$ for all $1 \leq i<j \leq k+1$; hence no model can be constructed satisfying card $_{\mathrm{S}, k}$.

Termination) It is easy to see that when the procedure recurses in Step 5 . the set of equalities and disequalities in $M$ without the cardinality constraints is satisfiable. Let $C$ be a set collecting the equivalence classes of $M^{*}$ and let $[t]_{M}$ denote the equivalence class of a term $t$. We argue that the splitting on the equality of $s$ and $t$ done at Step 5 decreases the size of the set

$$
E_{M}:=\left\{\left([u]_{M},[v]_{M}\right) \in C \times C \mid[u]_{M} \neq[v]_{M}, M \not \nvdash_{\mathrm{EUF}} u \not \approx v\right\}
$$

in other words, it decreases the number of equivalence classes that are pairwise not entailed to be disequal. In either branch of the split on $s \approx t$, no equivalence classes are created (although two existing ones are possibly merged), and $\left([s]_{M},[t]_{M}\right)$ is no longer an element of $E_{M}$ in the recursive call. When $E_{M}$ becomes empty, the procedure is guaranteed to terminate, since either more than $k$ equivalence classes are entailed to be distinct, in which case the procedure answers unsat, or there are at most $k$ equivalence classes, in which case the procedure answers sat.

Completeness) The procedure answers sat when the congruence closure $M^{*}$ contains no equality whose negation occurs in $M$, and either there is no positive cardinality literal in $M$, or $M^{*}$ has at most $k$ equivalence classes where $k$ is the smallest integer such that card $_{\mathrm{s}, k} \in M$. In either case, we can construct a model where $S$ is interpreted as a set of size $j$, with $j \leq k$ for all cards, $\in M$ and $j \geq k$ for all $\neg$ cards,,$k \in M$. If $j$ is greater than the number of equivalence classes in $M^{*}$, arbitrary new elements can be added to the domain of $S$ without affecting the satisfiability of the equalities and disequalities in $M$.

An immediate consequence of this lemma is that constraint satisfiability in FCC is decidable.

Proposition 2 The FCC-satisfiability of sets of $\Sigma_{\mathrm{FCC}}$-literals is decidable.

The completeness argument in Lemma 1 also suggests a constructive proof of the following result.

Proposition 3 Every satisfiable set of $\Sigma_{\mathrm{FCC}}$-literals has a finite model.

We point out that in the absence of cardinality constraints the decision procedure in Figure 4 reduces to the standard congruence closure procedure used to decide the satisfiability of constraints in EUF. SMT solvers supporting EUF have theory solvers that essentially implement that procedure.

6.2 Integration into $\operatorname{DPLL}\left(T_{1}, \ldots, T_{m}\right)$

Our decision procedure for FCC can be integrated into the $\operatorname{DPLL}\left(T_{1}, \ldots, T_{m}\right)$ framework by capitalizing on the existence of a theory solver for EUF $\left(T_{e}\right)$. We effectively extend such a solver modularly with facilities to reason about cardinality constraints as well. Since FCC is an extension of EUF, we now replace the latter with the former in the framework and make $T_{e}=\mathrm{FCC}$. Recall the strategy outlined in Figure 2 of Section 4.4 . In the following, we detail how the methods weak_effort and strong_effort of this strategy are implemented for FCC. 


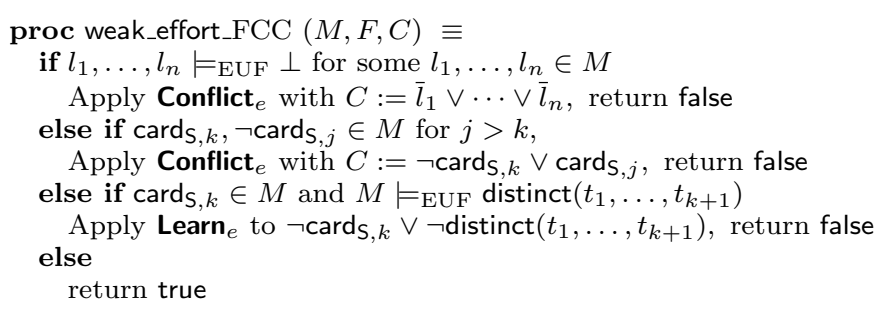

Fig. 5 Weak effort check for FCC.

For simplicity, we maintain the restriction for now that FCC contains a single uninterpreted sort S. Also, when convenient, we identify equivalence classes of terms with their representative terms.

\section{Weak Effort Check}

At weak effort, we recognize conflicting states of three different forms, outlined in Figure 5. First, if we are unable to construct a congruence closure for $M$ that is consistent with the disequalities from $M$, we identify a subset $\left\{l_{1}, \ldots, l_{n}\right\}$ of $M$ that is EUF-unsatisfiable and apply Conflict $_{e}$ to it. Second, if $M$ contains the conflicting

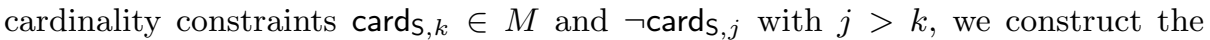
conflict clause $\neg$ cards, $k \vee$ cards, $_{s}$. Third, we may recognize cases when $M$ contains a literal of the form cards, $k$ while its other literals entails that $k+1$ terms $t_{1}, \ldots, t_{k+1}$ are pairwise disequal. In this case, we use Learn $_{e}$ to add the lemma $\neg$ cards, $k \vee$ $\neg$ distinct $\left(t_{1}, \ldots, t_{k+1}\right)$ to the current set $F$ of clauses, where $\operatorname{distinct}\left(t_{1}, \ldots, t_{k+1}\right)$ is shorthand for the conjunction of disequalities stating that the terms $t_{1}, \ldots, t_{k+1}$ are pairwise distinct ${ }^{3}$ We will refer to a lemma of this form as a clique lemma. We assume that this instance of Learn $_{e}$ is applied only when the resultant clause does not occur in $F$. In practice, this can be achieved either by maintaining a cache of learned clauses or by ensuring Propagate $e_{e}$ is applied to completion between each call. We apply Learn $_{e}$ because the constraint $\neg$ distinct $\left(t_{1}, \ldots, t_{k+1}\right)$ may contain literals not belonging to $F$. We could alternatively apply Conflict $_{e}$ to construct a conflict clause of form $\bar{l}_{1} \vee \ldots \vee \bar{l}_{n} \vee \neg$ cards,$k_{\mathrm{s}}$, where $\left\{l_{1}, \ldots, l_{n}\right\}$ is a subset of $M$ that entails distinct $\left(t_{1}, \ldots, t_{k+1}\right)$. However, we have found that in practice this is inefficient, as many different sets of literals can be found for essentially the same conflict.

Generating clique lemmas For the purposes of discovering and learning clique lemmas, we incrementally construct and maintain on the side a disequality graph $D$ for $\mathrm{S}$, whose vertices correspond to the equivalence classes of terms of sort $\mathrm{S}$ induced by the congruence closure of $M$, and whose edges represent disequalities in $M$ between terms in different equivalence classes. In this representation, a sufficient condition for discovering a conflict reduces to finding a $(k+1)$-clique in $D$. Now, even just checking for the presence of a $(k+1)$-clique in a $n$-vertex graph is too expensive in general-as this is an NP-complete problem [15]. For this reason, the weak effort check of our procedure uses an incomplete check for potential cliques.

\footnotetext{
${ }^{3}$ Note that $\neg$ card $_{\mathrm{S}, k} \vee \neg$ distinct $\left(t_{1}, \ldots, t_{k+1}\right)$ is a valid formula of FCC.
} 
This is done by partitioning the vertices of the graph into suitable subsets that we call regions. After defining regions formally, we explain below how we exploit them to discover clique-related conflicts efficiently in practice.

Definition 3 ( $k$-Region) Let $D=(V, E)$ be an undirected graph and let $R$ be a subset of $V$. For all vertices $v \in R$, let $\operatorname{ext}(v)$ be the number of edges between $v$ and vertices not in $R$. We say $R$ is a $k$-region of $D$ if for all $0<i \leq k$, the size of the set $\{v \mid v \in R, \operatorname{ext}(v) \geq i\}$ is smaller than $k-i$. A $k$-regionalization $\mathcal{R}_{D}$ of $D$ is a partition of $V$ into $k$-regions. We will refer to it simply as a regionalization when $k$ is understood or not important.

Regionalizations are useful for us because they facilitate the discovery of cliques.

Lemma 2 If $\mathcal{R}_{D}$ is a $k$-regionalization of a graph $D$ and $D$ contains a $k$-clique $C$, then all the vertices of $C$ reside in the same region of $\mathcal{R}_{D}$.

Proof: If $k \leq 1$, the statement is trivial. Otherwise, assume by contradiction $D$ contains $k$-clique $C=C_{1} \cup C_{2}$ for non-empty $C_{1}, C_{2}$ where, for some region $R$ of $\mathcal{R}_{D}, v \in R$ for all $v \in C_{1}$ and $v \notin R$ for all $v \in C_{2}$. Say $\left|C_{2}\right|=i$, and thus $\left|C_{1}\right|=k-i$. Since $C$ is a $k$-clique, $\operatorname{ext}(v)$ must be at least $i$ for all $v \in C_{1}$, contradicting the assumption that $R$ is a region.

Notice that any graph $D=(V, E)$ has a trivial regionalization, with just one region which contains all vertices in $V$.

Example 2 Consider the constraints $\left\{c_{1} \not \approx c_{2}, c_{2} \not c_{3}, c_{3} \not \approx c_{4}\right\}$, all over sort $\mathrm{S}$, and the partition $\left\{\left\{c_{1}, c_{2}\right\},\left\{c_{3}, c_{4}\right\}\right\}$. This partition is a 3 -regionalization in the disequality graph induced by this set, because a 3 -clique can span two regions only if it contains two vertices with interregional edges, and this partition only has one such edge. Adding the disequality $c_{2} \not c_{4}$ or $c_{1} \not \approx c_{4}$ breaks the regionalization invariant.

Let us examine how to maintain a $k$-regionalization in an (initially empty) evolving graph $D$, a data structure supporting the dynamic allocation of vertices and edges, as well as the merging of vertices. In our framework, where $D$ 's vertices correspond to equivalence classes of terms and edges to disequalities between them, these operations are triggered by operations performed on the data structure that stores the congruence closure $M^{*}$ of the current assignment $M$. In particular, a vertex $v_{e}$ is added to $D$ when a new equivalence class $e$ is created, which happens whenever a new term is added to $M$; an edge between the vertices $v_{1}$ and $v_{2}$ corresponding to equivalence classes $e_{1}$ and $e_{2}$ is added to $D$ when the disequation $t_{1} \not t_{2}$ is added to $M$, for some term $t_{1}$ in $e_{1}$ and $t_{2}$ in $e_{2}$; and two vertices are merged, in a single vertex that inherits their edges, when their corresponding equivalence classes are merged during the computation of $M^{*}$.

We maintain at all times a $(k+1)$-regionalization $\mathcal{R}_{D}$ of the graph $D$, where $k$ is the smallest integer such as cards, $\in M{ }^{4}$ As the graph $D$ is modified, it may be necessary to merge certain regions of the current within $\mathcal{R}_{D}$ to ensure the invariant in Definition 3 holds. The procedure fix_region from Figure 6 ensures that a set $R$ within $\mathcal{R}_{D}$ is a $k$-region by merging it as needed with another set

\footnotetext{
4 Recall that card $_{\mathrm{S}, k}$ states that sort $\mathrm{S}$ has at most $k$ elements.
} 


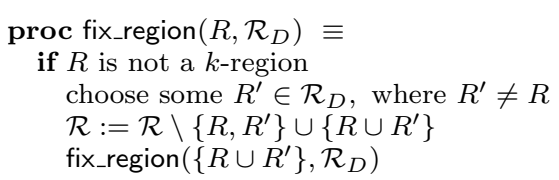

Fig. 6 The fix_region procedure. Ensures $R \in \mathcal{R}_{D}$ is a $k$-region by merging it with another $R^{\prime} \in \mathcal{R}_{D}$, and repeating this process recursively.

$R^{\prime}$ in $\mathcal{R}_{D}$, and repeating this process recursively until $R$ becomes a $k$-region. As a heuristic, we choose the $R^{\prime}$ with the highest density of interregional edges to $R$.

Assuming we have a regionalization $\mathcal{R}_{D}$ for graph $D$, here is how we construct a regionalization $\mathcal{R}_{D^{\prime}}$ for graph $D^{\prime}$ resulting from an addition to $M$. In the following, $\mathcal{R}(v)$ denotes the region in a regionalization $\mathcal{R}$ that contains the vertex $v$.

Adding Vertices: When a vertex $v$ is added to $D, \mathcal{R}_{D^{\prime}}$ is the result of adding the singleton region $\{v\}$ to $\mathcal{R}_{D}$.

Adding Edges: When we add an edge $\left(v_{1}, v_{2}\right)$ to $D$, we have that $\mathcal{R}_{D^{\prime}}=\mathcal{R}_{D}$ is still a partition of $V$. However, $\mathcal{R}_{D}\left(v_{1}\right)$ or $\mathcal{R}_{D}\left(v_{2}\right)$ may not be regions of $D^{\prime}$. We apply the procedure fix_region first to $\left(\mathcal{R}_{D}\left(v_{1}\right), \mathcal{R}_{D^{\prime}}\right)$ and then to $\left(\mathcal{R}_{D}\left(v_{2}\right), \mathcal{R}_{D^{\prime}}\right)$ to ensure that $\mathcal{R}_{D^{\prime}}$ is a regionalization.

Merging Vertices: When a vertex $v_{1}$ is merged with another vertex $v_{2}$ in $D$, we have that $D^{\prime}$ is a quotient graph of $D$, that is, $D^{\prime}$ contains a new vertex, call it $u$, connected to all vertices that are connected to either $v_{1}$ or $v_{2}$ in $D$. If $\mathcal{R}_{D}\left(v_{1}\right)$ is equal to $\mathcal{R}_{D}\left(v_{2}\right)$, let $R$ be $\left(\mathcal{R}_{D}\left(v_{1}\right) \cup\{u\}\right) \backslash\left\{v_{1}, v_{2}\right\}$. Then $\mathcal{R}_{D^{\prime}}$ is equal to $\left(\mathcal{R}_{D} \cup R\right) \backslash\left\{\mathcal{R}_{D}\left(v_{1}\right)\right\}$. To ensure $\mathcal{R}_{D^{\prime}}$ is a regionalization, we apply fix_region to $\left(R, \mathcal{R}_{D^{\prime}}\right)$. If $\mathcal{R}_{D}\left(v_{1}\right)$ is not equal to $\mathcal{R}_{D}\left(v_{2}\right)$, let $\left\{v_{i}, v_{j}\right\}=\left\{v_{1}, v_{2}\right\}$, $R_{i}=\left(\mathcal{R}_{D}\left(v_{i}\right) \cup\{u\}\right) \backslash\left\{v_{i}\right\}$, and $R_{j}=\mathcal{R}_{D}\left(v_{j}\right) \backslash\left\{v_{j}\right\}$. Then, $\mathcal{R}_{D^{\prime}}$ is equal to $\left(\mathcal{R}_{D} \cup\left\{R_{i}, R_{j}\right\}\right) \backslash\left\{\mathcal{R}_{D}\left(v_{1}\right), \mathcal{R}_{D}\left(v_{2}\right)\right\}$. We apply fix_region to $\left(R_{i}, \mathcal{R}_{D^{\prime}}\right)$ and subsequently to $\left(R_{j}, \mathcal{R}_{D^{\prime}}\right)$.

Additionally, when card $_{\mathrm{s}, k^{\prime}}$ is asserted for $k^{\prime}<k$, we discard the $(k+1)$ regionalization and rebuild a $\left(k^{\prime}+1\right)$-regionalization.

Given a $(k+1)$-regionalization $\mathcal{R}_{D}$ of $D$, we will call each region in $\mathcal{R}_{D}$ with at least $k+1$ vertices a large region, and all others small regions. For the purposes of efficiently discovering $(k+1)$-cliques during weak effort checks, we maintain a watched set of $k+1$ vertices for each large region $R$ in $\mathcal{R}_{D}$, which we will write as $w(R)$. This set is incrementally updated when vertices are added or removed from regions, and when regions are combined. If there exists a large region $R$ in $\mathcal{R}_{D}$ where each vertex in $w(R)$ is connected, then we add the clique lemma $\neg$ cards, $k \vee$

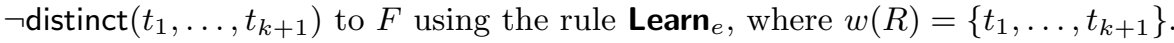

\section{Strong Effort Check}

Recall from Section 4.4 that a strong effort check must determine that the current set of constraints is consistent, or otherwise report a conflict or lemma. The strong effort check of the FCC solver is given in Figure 77. If cards, $k \in M$ for some (minimal) $k$, and there are more than $k$ equivalence class of sort $\mathrm{S}$ in the congruence closure of $M$, then we choose two equivalence class representatives $t_{i}$ and $t_{j}$ and

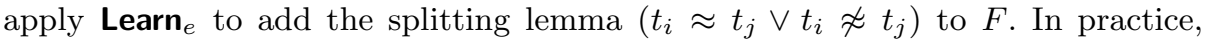




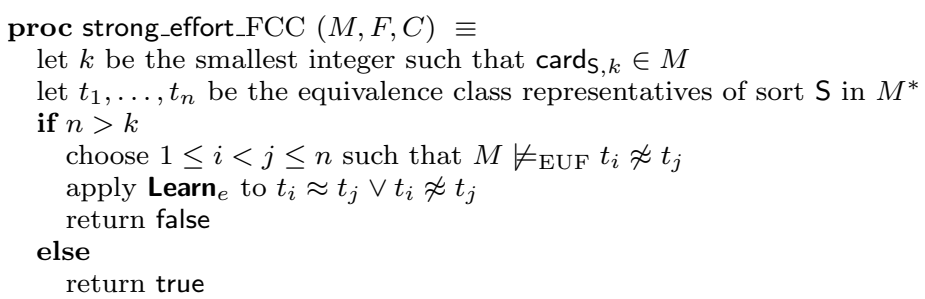

Fig. 7 Strong effort check for FCC.

we also insist that future applications of Decide on the atom $t_{i} \approx t_{j}$ should be invoked with positive polarity. If the number of equivalence classes is less than or equal to $k$, then the procedure returns true, indicating that $M$ is FCC-satisfiable.

The choice of $t_{i}$ and $t_{j}$ is guided by the watched set of vertices within regions. In particular, if there is a large region $R$ in $\mathcal{R}_{D}$, we know that $w(R)$ does not form a clique. We choose $t_{i}, t_{j}$ to be two vertices from $w(R)$ that are not connected in $D$. Otherwise, if there are no large regions in $\mathcal{R}_{D}$ and there are more than $k$ vertices in $D$, then there must be at least two small regions. We select two regions $R_{i}$ and $R_{j}$ based on a heuristic (namely, the maximum density of interregional edges), combine them into a new region $R_{i} \cup R_{j}$, apply fix_region to $R_{i} \cup R_{j}$, and repeat.

We illustrate the operation of the FCC solver with a couple of examples.

Example 3 Consider the constraints $\left\{a \approx f(b), b \approx f(c), a \not z b, b \not c, \operatorname{card}_{\mathrm{S}, 2}\right\}$ where all terms are over the single sort S. First, the FCC solver computes the congruence $\{\{a, f(b)\},\{b, f(c)\},\{c\}\}$. Using $a, b, c$ as the representatives, the solver builds the disequality graph with edges $\{(a, b),(b, c)\}$. Since cards, limits the size of $\mathrm{S}$ to at most 2 , the solver generates the lemma $a \approx c \vee a \not c$. Adding the constraint $a \approx c$ produces no conflicts and allows the FCC solver to answer "satisfiable".

Example 4 Consider the constraints $\left\{c_{1} \approx c, c_{4} \approx c, c_{1} \not c_{2}, c_{2} \not \approx c_{3}, c_{3} \not \approx\right.$ $\left.c_{4}, \mathrm{card}_{\mathrm{S}, 2}\right\}$ with all constants of sort $\mathrm{S}$. The corresponding disequality graph for these constraints contains a clique of size 3. By discovering that clique, the FCC solver can conclude that it is impossible to shrink the model to 2 elements, and hence reports a clique lemma of the form $\neg$ distinct $\left(c_{1}, c_{2}, c_{3}\right) \vee \neg$ card $_{\mathrm{S}, 2}$.

Because of congruence constraints, guesses on merge lemmas may sometimes lead to inconsistencies when constructing the congruence closure, unless we compute and propagate all entailed disequalities - which is usually not done, for efficiency. This is demonstrated in the following example.

Example 5 Consider the constraints $\left\{c_{3} \approx f\left(c_{1}\right), c_{4} \approx f\left(c_{2}\right), c_{3} \not \approx c_{4}, \operatorname{card}_{\mathrm{s}, 2}\right\}$ where all the terms have sort S. Unless the EUF subsolver propagates the entailed literal $c_{1} \not c_{2}$, the FCC solver will construct the disequality graph $(V, E)=$ $\left(\left\{c_{1}, c_{2}, c_{3}, c_{4}\right\},\left\{\left(c_{3}, c_{4}\right)\right\}\right)$ for S. Say we decide to apply Learn on $\left(c_{1} \approx c_{2} \vee c_{1} \not\right.$ $\left.c_{2}\right)$, and then the literal $c_{1} \approx c_{2}$ is added to our set of constraints. The subset $\left\{c_{3} \approx f\left(c_{1}\right), c_{4} \approx f\left(c_{2}\right), c_{3} \not c_{4}, c_{1} \approx c_{2}\right\}$ will then be found unsatisfiable by congruence closure. In contrast, adding the equalities $c_{1} \approx c_{3}$ and $c_{2} \approx c_{4}$ to our set will produce a model of the required cardinality. 
We now state the correctness of our FCC procedure as integrated in the $\operatorname{DPLL}\left(T_{1}, \ldots, T_{m}\right)$ framework. In the following, we let check ${ }_{F C C}$ denote the strategy that applies the rules of $\operatorname{DPLL}\left(T_{\mathrm{FCC}}\right)$ according to Figure 2, with the weak and strong effort checks described in this section.

Theorem 3 check $\mathrm{FCC}_{\mathrm{FC}}$ is a sound, complete and terminating strategy for every set of ground clauses $F_{0}$.

Proof: Notice that the weak and strong effort methods in this section legally apply $\operatorname{DPLL}\left(T_{1}, \ldots, T_{m}\right)$ rules, that is, they apply Conflict Cnly $_{e}$ to clauses whose negated literals imply a contradiction and Learn $_{e}$ to clauses that hold in all models. We follow the three requirements for weak and strong effort checks as described in Proposition 1 .

To show the first point, the only literals introduced by applications of Learn $_{e}$ (call them $L_{\mathrm{FCC}}$ ) are equalities and disequalities between terms occurring in $F_{0}$. Clearly $L_{\mathrm{FCC}}$ is finite. To show the second point, the weak and strong effort methods in this section false only when they apply at least one rule. To show the third point, strong_effort_FCC $(M, F, C)$ returns true only when the congruence closure of $M$ contains $k$ or fewer equivalence classes for all cards, $k \in M$. In such states, we are guaranteed that $M$ is satisfiable in FCC.

\subsection{Establishing Finite Cardinalities}

We have now shown that a theory solver for FCC can be integrated into the $\operatorname{DPLL}\left(T_{1}, \ldots, T_{m}\right)$ architecture with support for eager conflict detection through the use of weak effort checks. In this section, we show an approach that makes use of this solver for answering the following problem: given an input $F$, find the smallest integer $n>0$ such that $F \wedge$ card $_{\mathrm{S}, n}$ is satisfiable.

A straightforward scheme for solving this problem is the following. First, use the solver to determine if $F \wedge$ cards, $1_{\mathrm{S}}$ is satisfiable, and answer satisfiable if so. If this

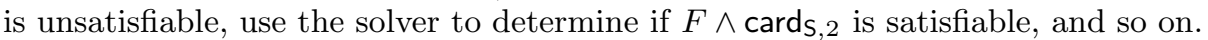
Due to Proposition 3 this process is guaranteed to terminate when $F$ is satisfiable. A clear disadvantage of this scheme is that, in the absence of conflict analysis, it diverges when $F$ is unsatisfiable. This section describes an alternative approach that overcomes this limitation. At a high level, our approach modifies the weak effort check of the FCC solver by introducing splits on cardinality constraints (card $_{\mathrm{S}, k} \vee \neg \operatorname{card}_{\mathrm{S}, k}$ ), and deciding upon literals of the $\operatorname{card}_{\mathrm{S}, k}$ for the minimal feasible $k$. Before formally defining this approach, we discuss a generalization that is applicable to signatures with multiple uninterpreted sorts.

\section{Extension to Multiple Sorts}

Consider the case when our signature $\Sigma$ contains multiple sorts $\mathrm{S}_{1}, \ldots, \mathrm{S}_{n}$. Given a set of input clauses $F$, we wish to determine that either $F$ is unsatisfiable, or find a tuple $\left(k_{1}, \ldots, k_{n}\right)$ such that $F \wedge \operatorname{card}_{\mathrm{S}_{1}, k_{1}} \wedge \ldots \wedge \operatorname{card}_{\mathrm{S}_{n}, k_{n}}$ is satisfiable. To find such a tuple, a challenge is to devise a strategy that is fair. As an illustrative example, consider the formula $(c \not \approx \vee \vee)$, where $c$ and $d$ are constants of sort $\mathrm{S}_{1}$, 


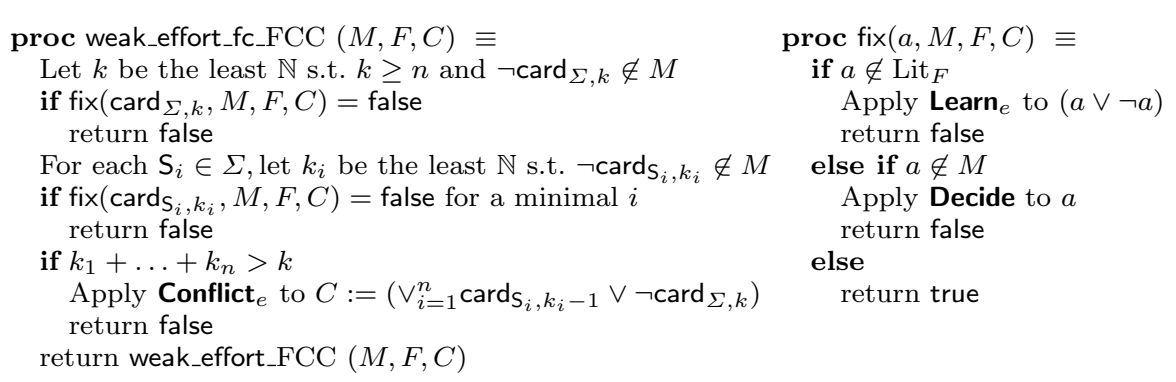

Fig. 8 A version of the weak effort check procedure of the FCC solver that fixes the cardinality of uninterpreted sorts $\left\{\mathrm{S}_{1}, \ldots, \mathrm{S}_{n}\right\}$ in signature $\Sigma$ according to a fair strategy.

and the formula $\varphi$ does not have a model where $S_{2}$ is interpreted as a finite set 5 Clearly this formula has a model where the cardinality of sorts $S_{1}$ and $S_{2}$ are 2 and 1 respectively. However, in the absence of a fair strategy, a naive approach could search for models of size $(1,1),(1,2),(1,3)$, and so on, ad infinitum.

To devise a strategy for finite model finding that is fair in the presence of multiple sorts, we extend the signature $\Sigma$ of FCC to include signature cardinality constraints $\operatorname{card}_{\Sigma, k}$, constants of sort Bool for each integer $k>0$. Let $\Sigma$ be a signature containing uninterpreted sorts $\mathrm{S}_{1}, \ldots, \mathrm{S}_{n}$. Let $\mathcal{I}$ be a $\Sigma$-interpretation that interprets sort $\mathrm{S}_{i} \in \Sigma$ as a set of size $k_{i}$ for $1 \leq i \leq n$. Then, $\mathcal{I}$ satisfies $\operatorname{card}_{\Sigma, k}$ if and only if $k_{1}+\ldots+k_{n} \leq k$.

Figure 8 gives an extension of the weak effort check of the FCC solver that introduces cardinality constraints for the purposes of finding small models. In detail, we first find the minimal natural number $k$ such that the literal $\neg \operatorname{card}_{\Sigma, k}$ does not occur in $M$. Using the sub-routine fix, if the atom card ${ }_{\Sigma, k}$ does not occur

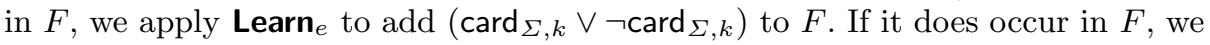
apply Decide to $\operatorname{card}_{\Sigma, k}$. We then do the same for each of the uninterpreted sorts $\mathrm{S}_{1}, \ldots, \mathrm{S}_{n}$ in our signature. If these steps do not apply a rule, then $M$ contains the literals $\operatorname{card}_{\Sigma, k}$ and $\neg \operatorname{card}_{\mathrm{S}_{i}, \ell}$ for each $1 \leq \ell<k_{i}, i=1, \ldots, n$. We then check if $\operatorname{card}_{\Sigma, k}$ is in conflict with the negatively asserted cardinality constraints. In particular, $k_{1}+\ldots+k_{n}>k$, we return a conflict of the form $\left(\operatorname{card}_{\mathrm{s}_{1}, k_{1}-1} \vee\right.$ $\left.\ldots \vee \operatorname{card}_{\mathrm{S}_{n}, k_{n}-1} \vee \neg \operatorname{card}_{\Sigma, k}\right)$, where we write $\operatorname{card}_{\mathrm{S}_{i}, k_{i}-1}$ to denote a cardinality constraint when $k_{i}>1$ and $\perp$ if $k_{i}=1$. Otherwise, we apply the original weak effort check of the FCC solver from Figure 5.

Let fixed-cardinality check $\mathrm{FCC}_{\mathrm{C}}$ be the strategy that applies the rules of DPLL $\left(T_{\mathrm{FCC}}\right)$ according to Figure 2 where the weak effort check is the one from Figure 8 , and the strong effort check is the one from Figure 7. This strategy maintains the following invariant.

Proposition 4 Given a signature $\Sigma$ containing uninterpreted sorts $\mathrm{S}_{1}, \ldots, \mathrm{S}_{n}$, for each execution of fixed-cardinality check ${ }_{\mathrm{FCC}}$ ending in $\langle M, F, C\rangle$, either $M$ contains no decision points, or $M$ is of the form $N \bullet \operatorname{card}_{\Sigma, k} M_{0}\left(\bullet \operatorname{card}_{\mathrm{S}_{1}, k_{1}} M_{1}\right)$ $\cdots \quad\left(\bullet \operatorname{card}_{\mathrm{s}_{m}, k_{m}} M_{m}\right) N^{\prime}$, for some $m, 0 \leq m \leq n$, where $N, M_{0}, \ldots, M_{m}$ contain no decision points, $N^{\prime}$ contains no decision points if $m<n, \neg \operatorname{card}_{\Sigma, j} \prec_{M} \operatorname{card}_{\Sigma, k}$ for each $n \leq j<k$, and $\neg \operatorname{card}_{s_{i}, j} \prec_{M}$ card $_{\mathrm{S}_{i}, k_{i}}$ for each $1 \leq i \leq m, 1 \leq j<k_{i}$.

\footnotetext{
5 Observe that $\varphi$ must contain universal quantifiers for this to be the case.
} 
In other words, using the strategy fixed-cardinality check ${ }_{\mathrm{FCC}}$, minimal positive cardinality literals are the first decision literals in satisfying assignments. This invariant follows directly from definition of the method given in Figure 8.

Theorem 4 Fixed-cardinality check $\mathrm{FCC}_{\mathrm{F}}$ is a sound, complete and terminating strategy for every set of ground clauses $F$.

Proof: Assume our signature $\Sigma$ contains uninterpreted sorts $\mathrm{S}_{1}, \ldots, \mathrm{S}_{n}$. Note that the weak effort check method in Figure 8 extends our original weak effort check while additionally applying only legal applications of $\operatorname{DPLL}\left(T_{1}, \ldots, T_{m}\right)$ rules,

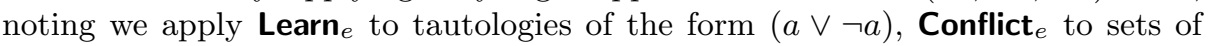
literals that are collectively inconsistent according to our extension of FCC, and Decide to literals whose atom does not occur in $M$. To show this strategy is sound, complete, and terminating, we again follow the three requirements for weak and strong effort checks as given in Proposition 1 .

To show the first point, we must show that the set $L_{\mathrm{FCC}}$ of literals introduced by applications of Learn $_{e}$ is finite. For each $1 \leq i \leq n$, let $k_{i}$ be smallest integer greater than the number of terms of sort $\mathrm{S}_{i}$ in $F$, and such that the literal $\neg \operatorname{card}_{\mathrm{s}_{i}, k_{i}}$ does not occur in $F$. Let $k$ be the smallest integer greater than $k_{1}+\ldots+k_{n}$, and such that the literal $\neg \operatorname{card}_{\Sigma, k}$ does not occur in $F$. We claim that the set of literals introduced by applications of Learn $_{e}$, call them $L_{\mathrm{FCC}}^{f c}$, are a subset of the set of all equalities and disequalities between terms from $F$, the literals of

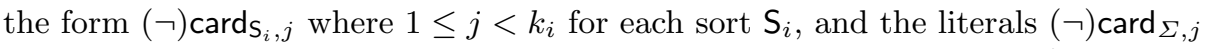
where $n \leq j<k$. First, only equalities and disequalities between terms from $F$ are introduced by applications of Learn $_{e}$ for the same reason as in Theorem 3 . Second,

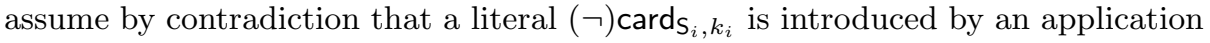

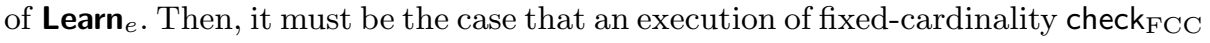
results in a state where $\neg \operatorname{card}_{S_{i}, k_{i}-1} \in M$. For this to be the case, $\neg$ cards $_{\mathrm{S}_{i}, k_{i}-1}$ must be added to $M$ by Propagate $_{e}$ or Backjump. In either case, there must exist a set of literals $l_{1}, \ldots, l_{n}$ from $F$ such that $l_{1}, \ldots, l_{n} \models_{\text {FCC }} \neg$ card $_{s_{i}, k_{i}-1}$. By our selection of $k_{i}$ this is a contradiction since there must be at least $k_{i}$ terms of sort $\mathrm{S}_{i}$ in $F$ for this to be the case. Third, for similar reasons, by our selection of $k$, a literal $(\neg)_{\operatorname{card}_{\Sigma, k}}$ cannot be introduced by an application of Learn $_{e}$ unless there exists an execution of fixed-cardinality check ${ }_{\mathrm{FCC}}$ resulting in a state where $\neg \operatorname{card}_{\mathrm{S}_{i}, j} \in M$ for some $\mathrm{S}_{i}$ where $j \geq k_{i}$. This cannot be the case for the reasons mentioned above.

To show the second point, weak effort check method in Figure 8 returns false only when it applies a rule.

To show the third point, the strong effort check of fixed-cardinality check $_{\text {FCC }}$ is the same as the strong effort check in check ${ }_{\mathrm{FCC}}$ and thus this holds for the same reason as in the proof of Theorem 3 .

Combining the results of Theorem 4 and Proposition 4 , given as input a set of ground $\Sigma$-clauses $F$, fixed-cardinality check ${ }_{\mathrm{FCC}}$ will terminate either in $(i)$ a fail state, establishing that $F$ is unsatisfiable, or $(i i)$ a state $\langle M, F$, no $\rangle$ where $M$ contains card $_{S_{i}, k_{i}}$ for each uninterpreted sort $\mathrm{S}_{i}$ in $\Sigma$, establishing that $F$ is satisfied by a (finite) model.

For the remainder of the paper, we assume that Step 1 of our finite model finding procedure in Figure 3 uses fixed-cardinality check ${ }_{\mathrm{FCC}}$ for finding satisfying assignments $M$. 


\section{Constructing Candidate Models}

We now focus our attention to Step 2 of procedure FM-Solve $e_{\mathcal{H}}$ from Figure 3 . which attempts to constructs models $\mathcal{M}$ of satisfying assignments $M$ for the input clause set $F$. We refer to $\mathcal{M}$ as a candidate model. Note that the assignment $M$ computed by the procedure may contain occurrences of proxy variables $a$ for quantified formulas $\forall \mathbf{x} \varphi$ with the variables in $\mathbf{x}$ ranging over uninterpreted or finite sorts. Recall that those formulas are stored in the input set $A$ in equivalences of the form $a \Leftrightarrow \forall \mathbf{x} \varphi$. The goal of the procedure is to construct $\mathcal{M}$ so that it satisfies not just $M$ but also all its active quantified formulas (those whose proxy variable $a$ occurs positively in $M$ ). The reason is that such a model witnesses the $T$-satisfiability of $F \cup A$.

To discuss the model construction we focus on the variables and the uninterpreted sorts and function symbols of $\Sigma$, since the interpretation of the other sorts and function symbols is fixed by the theory. We construct a candidate model $\mathcal{M}$ by associating each uninterpreted sort $S$ with a finite set $\mathbf{V}_{S}$ of domain elements (i.e., $\left.S^{\mathcal{M}}=\mathbf{V}_{S}\right)$. Contrary to other model finding approaches, which use fresh symbols as domain elements, we use the equivalence classes of $M^{*}$ or, rather, representative terms for these classes. All interpreted sorts are interpreted in $\mathcal{M}$ as usual. We extend $M^{*}$ to another $T$-satisfiable set, call it $M_{c}^{*}$, such that the representative of each equivalence class of interpreted sort $S_{i}$ in $M_{c}^{*}$ is a value from $S_{i}^{\mathcal{M}}$. Such an extension is always possible since $M$ is $T$-satisfiable.

We then associate each uninterpreted function $f$ of sort $S_{1} \times \ldots \times S_{n} \rightarrow S$ to a function $f^{\mathcal{M}}$ from $S_{1}^{\mathcal{M}} \times \cdots \times S_{n}^{\mathcal{M}}$ to $S^{\mathcal{M}}$. We construct this function based on the literals in $M$ that contain $f$. For instance, if $M$ contains $f(c) \approx b$, then $f^{\mathcal{M}}$ is defined so that it maps the interpretation of $c$ to the interpretation of $b$. Using those equalities typically produces only a partial definition for $f$. To complete it, one can use arbitrary output values for the missing input tuples. We describe choices for doing so in the following.

Concretely, we represent candidate $\Sigma$-models with the following data structure.

Definition 4 (Defining map) Let $f: S_{1} \times \cdots \times S_{n} \rightarrow S$ be an uninterpreted function symbol of $\Sigma$ and let $y_{1}, \ldots, y_{n}$ be distinct fresh variables of respective sort $S_{1}, \ldots, S_{n}$. A defining map for $f$ is a finite set $\Delta_{f}$ of well-sorted (directed) equations of the form $f\left(t_{1}, \ldots, t_{n}\right) \approx v$ with $v \in S^{\mathcal{M}}$ and $t_{i} \in\left\{y_{i}\right\} \cup S_{i}^{\mathcal{M}}$ for $i=1, \ldots, n$, satisfying the following requirements.

1. If $s_{1} \approx v_{1}, s_{2} \approx v_{2} \in \Delta_{f}$ with $s_{1} \neq s_{2}$ and $s_{1}$ and $s_{2}$ have an mgu $\sigma$, then

(a) $\sigma$ is non-empty, and

(b) $s_{1} \sigma \approx v \in \Delta_{f}$ for some $v$.

2. $f\left(y_{1}, \ldots, y_{n}\right) \approx v \in \Delta_{f}$ for some $v$.

A $\Sigma$-map is a set $\Delta=\bigcup_{f \in \Sigma^{\mathrm{f}}} \Delta_{f}$ where each $\Delta_{f}$ is a defining map for $f$.

For the rest of this section, we will use letters $y, y_{1}, y_{2}, \ldots$ to denote variables and $c, c_{1}, c_{2}, \ldots$ to denote constant symbols.

Example 6 The set $\left\{f\left(c_{1}, y_{2}\right) \approx c_{2}, f\left(y_{1}, c_{2}\right) \approx c_{1}, f\left(c_{1}, c_{2}\right) \approx c_{3}, f\left(y_{1}, y_{2}\right) \approx c_{3}\right\}$ is a defining map for $f$. Notice that $f\left(c_{1}, y_{2}\right)$ and $f\left(y_{1}, c_{2}\right)$ have mgu $\left\{y_{1} \mapsto c_{1}, y_{2} \mapsto\right.$ $\left.c_{2}\right\}$. As required in point 1 , this mgu is non-empty and an equality of the form $f\left(c_{1}, c_{2}\right) \approx v$ also occurs in this set. 
Input: A satisfying assignment $M$ and a set $U \subseteq \mathbf{T}_{M}$

Output: A $\Sigma$-map $\Delta$

1. For each uninterpreted function symbol $f: S_{1} \times \cdots \times S_{n} \rightarrow S$ of $\Sigma$,

(a) Let $D_{1}=\left\{f\left(\mathcal{V}_{M}\left(t_{1}\right), \ldots, \mathcal{V}_{M}\left(t_{n}\right)\right) \approx \mathcal{V}_{M}(t) \mid t \in \mathbf{T}_{M}, t=f\left(t_{1}, \ldots, t_{n}\right)\right\}$

(b) Let $D_{2}=\left\{f\left(t_{1}, \ldots, t_{n}\right)^{\forall} \approx \mathcal{V}_{M}(t) \mid t \in U, t=f\left(t_{1}, \ldots, t_{n}\right)\right\}$

(c) Let $\Delta_{f}=D_{1} \cup D_{2}$ and let $\left\{t_{i} \approx v_{i}\right\}_{0 \leq i \leq m}$ be an arbitrary enumeration of $\Delta_{f}$. For all $t_{i} \approx v_{i}, t_{j} \approx v_{j}$ with $i<j$ that are unifiable with mgu $\sigma$, if $t_{i} \sigma$ does not already occur as a left-hand side in $\Delta_{f}$, add $t_{i} \sigma \approx v_{i}$ to $\Delta_{f}$.

(d) If $f\left(y_{1}, \ldots, y_{n}\right)$ does not occur as a left-hand side in $\Delta_{f}$, add $f\left(y_{1}, \ldots, y_{n}\right) \approx v$ for some arbitrary value $v \in S^{\mathcal{M}}$.

2. Let $\Delta=\bigcup_{f \in \Sigma} \Delta_{f}$

Fig. 9 Model construction procedure.

By construction of $\Delta$, every flat term, a $\Sigma$-term $t=f\left(v_{1}, \ldots, v_{n}\right)$ has exactly one most specific generalization $s$ among the left-hand sides of the equalities in $\Delta_{f}$, where $s$ is a generalization of $t$ if $t=s \sigma$ for some substitution $\sigma$, and $s$ is more specific than $s^{\prime}$ if $s^{\prime}$ is a generalization of $s$. The existence of this generalization is guaranteed by Point 2 in the definition above; its uniqueness by Point 1 . The value of $t$ in $\Delta$ is the value $v$ in the (unique) equality $s \approx v \in \Delta_{f}$. Thus, a $\Sigma$-map $\Delta$ represents a normal model $\mathcal{M}$ where each uninterpreted sort $S$ is interpreted as the term set $\mathbf{V}_{S}$ and each uninterpreted function symbol $f: S_{1} \times \cdots \times S_{n} \rightarrow S$ is interpreted as the function $f^{\mathcal{M}}$ mapping every $\left(v_{1}, \ldots, v_{n}\right) \in S_{1}^{\mathcal{M}} \times \cdots \times S_{n}^{\mathcal{M}}$ to the value of $f\left(v_{1}, \ldots, v_{n}\right)$ in $\Delta 6^{6}$

\section{Model Construction Procedure}

We now describe a procedure for constructing $\Sigma$-maps from satisfying assignments. In particular, we describe a parametrized method for completing the partial definitions (of uninterpreted functions) induced by an assignment $M$.

Let $M$ be an assignment. Recall that if $M$ is $T$-satisfiable, it is satisfied by a normal model, that is, a model that interprets each uninterpreted sort $S$ as the set $\mathbf{V}_{S}$ consisting of a representative term for each equivalence class of (the extension of) $M$ 's congruence closure $M_{c}^{*}$. For each term $t$, we write $\mathcal{V}_{M}(t)$ to denote the representative of $t$ 's equivalence class in $M_{c}^{*}$.

For every uninterpreted function symbol $f: S_{1} \times \cdots \times S_{n} \rightarrow S$ in $\Sigma$, we fix $n$ distinct fresh variables $y_{1}, \ldots, y_{n}$ of respective sort $S_{1} \ldots, S_{n}$. To each uninterpreted sort $S$, we associate a distinguished ground $\Sigma$-term $e^{S}$, which we will write as $e$ when $S$ is understood. This ground term guides the selection of default values of the interpretation of uninterpreted function symbols in our model construction procedure, based on the following operation. For a ground $\Sigma$-term $f\left(t_{1}, \ldots, t_{n}\right)$, we denote by $f\left(t_{1}, \ldots, t_{n}\right)^{\forall}$ the term $f\left(u_{1}, \ldots, u_{n}\right)$ where $u_{i}=y_{i}$ if $t_{i}=e$, and $u_{i}=\mathcal{V}_{M}\left(t_{i}\right)$ otherwise, for $i=1, \ldots, n$.

6 More precisely, a $\Sigma$-map represents a family of normal models which differ only over the variables and the interpreted symbols of $\Sigma$. 
The non-deterministic procedure described in Figure 9 constructs a $\Sigma$-map from $M$ and a subset $U$ of the set of terms $\mathbf{T}_{M}$ occurring in $M$. The subset $U$ determines which terms will be used as the basis for default values of function interpretations. For example, let $\mathcal{M}$ be a normal model induced by the defining map constructed by the procedure in Figure 9 for some $U$. If $f(e, e) \in U$, then the default value of $f$ in $\mathcal{M}$ is the value of $f(e, e)$ in $\mathcal{M}$. In our implementation of the procedure, we choose the set $U$ to be the entire set $\mathbf{T}_{M}$, although other choices are possible.

Example 7 Consider an assignment $M$ with the following constraints

$$
\left\{c_{1} \approx f\left(c_{2}, e\right), c_{3} \approx f\left(c_{4}, c_{6}\right), c_{3} \approx f\left(e, c_{4}\right), c_{6} \approx f\left(c_{2}, c_{5}\right), c_{2} \approx c_{5}, c_{4} \approx f(e, e)\right\}
$$

where all terms are of uninterpreted sort S. The equivalence classes in the congruence closure of $M$ are

$$
\left\{c_{1}, f\left(c_{2}, e\right)\right\},\left\{c_{2}, c_{5}\right\},\left\{c_{3}, f\left(c_{4}, c_{6}\right), f\left(e, c_{4}\right)\right\},\left\{c_{4}, f(e, e)\right\},\left\{c_{6}, f\left(c_{2}, c_{5}\right)\right\},\{e\} .
$$

Let $\mathbf{V}_{S}=\left\{c_{1}, c_{2}, c_{3}, c_{4}, c_{6}, e\right\}$ and $U=\left\{f\left(c_{2}, e\right), f\left(e, c_{4}\right), f(e, e)\right\}$. Following the procedure to construct the defining map $\Delta_{f}$, we let:

$$
\begin{aligned}
& D_{1}=\left\{f\left(c_{2}, e\right) \approx c_{1}, f\left(c_{4}, c_{6}\right) \approx c_{3}, f\left(c_{2}, c_{2}\right) \approx c_{6}, f(e, e) \approx c_{4}\right\} \\
& D_{2}=\left\{f\left(c_{2}, y_{2}\right) \approx c_{1}, f\left(y_{1}, c_{4}\right) \approx c_{3}, f\left(y_{1}, y_{2}\right) \approx c_{4}\right\} \\
& \Delta_{f}=D_{1} \cup D_{2}
\end{aligned}
$$

Since $f\left(c_{2}, y_{2}\right)$ and $f\left(y_{1}, c_{4}\right)$ are unifiable with $\sigma=\left\{y_{1} \mapsto c_{2}, y_{2} \mapsto c_{4}\right\}$, and $f\left(c_{2}, c_{4}\right)$ is not in $\Delta_{f}$, we add the equality $f\left(c_{2}, c_{4}\right) \approx c_{1}$ (alternatively, $f\left(c_{2}, c_{4}\right) \approx$ $\left.c_{3}\right)$ to $\Delta_{f}$. Finally, since $f\left(y_{1}, y_{2}\right)$ is already in $\Delta_{f}$, this gives us the set

$$
\begin{aligned}
\Delta_{f}= & \left\{f\left(c_{2}, e\right) \approx c_{1}, f\left(c_{4}, c_{6}\right) \approx c_{3}, f\left(c_{2}, c_{2}\right) \approx c_{6}, f(e, e) \approx c_{4},\right. \\
& \left.f\left(c_{2}, y_{2}\right) \approx c_{1}, f\left(y_{1}, c_{4}\right) \approx c_{3}, f\left(y_{1}, y_{2}\right) \approx c_{4}, f\left(c_{2}, c_{4}\right) \approx c_{1}\right\}
\end{aligned}
$$

which is a complete definition for $f$. Notice that a different selection of $U$ would have led to a different construction for $\Delta_{f}$. Let $\mathcal{M}$ be the normal model induced by a $\Delta$ containing $\Delta_{f}$. We have that, for instance, $\mathcal{M} \llbracket f\left(c_{2}, c_{3}\right) \rrbracket=c_{1}$ since $f\left(c_{2}, y_{2}\right) \approx$ $c_{1} \in \Delta_{f}$ and $f\left(c_{2}, y_{2}\right)$ is the most specific generalization of $f\left(c_{2}, c_{3}\right)$ among the left-hand sides of equalities in $\Delta_{f}$. Similarly, we have that $\mathcal{M} \llbracket f\left(c_{6}, c_{4}\right) \rrbracket=c_{3}$ and $\mathcal{M} \llbracket f\left(c_{3}, c_{3}\right) \rrbracket=c_{4}$.

Proposition 5 Let $M$ be a T-satisfiable assignment containing only uninterpreted function symbols over uninterpreted sorts. The set $\Delta$ constructed by the procedure in Figure 9 is a $\Sigma$-map. Moreover, the normal model $\mathcal{M}$ represented by $\Delta$ satisfies $M$.

Proof: To show that $\Delta$ is a $\Sigma$-map, we show that $\Sigma_{f}$ is a defining map for each function symbol $f$ of $\Sigma$. Step 1 (c) of the procedure ensures that Point 1(b) of Definition 4 is met for all pairs of equalities in $\Delta_{f}$, while Step 1 (d) makes sure that Point 2 is met. We prove by contradiction that Point 1 (a) of Definition 4 also holds for $\Delta_{f}$. Assume that $t \approx v_{1}, t \approx v_{2} \in \Delta_{f}$ with $v_{1} \neq v_{2}$. Due to our construction, both $t \approx v_{1}$ and $t \approx v_{2}$ are in $D_{1} \cup D_{2}$. Thus, there must exist terms $t=f\left(t_{1}, \ldots, t_{n}\right)$ and $s=f\left(s_{1}, \ldots, s_{n}\right)$ in $\mathbf{T}_{M}$ such that $\mathcal{V}_{M}\left(t_{1}\right)=\mathcal{V}_{M}\left(s_{1}\right), \ldots$, 
$\mathcal{V}_{M}\left(t_{n}\right)=\mathcal{V}_{M}\left(s_{n}\right)$ and $\mathcal{V}_{M}(t)=v_{1} \neq v_{2}=\mathcal{V}_{M}(s)$, contradicting our assumption that $M$ is a (consistent) satisfying assignment. Thus, $\Delta_{f}$ is a defining map for all $f \in \Sigma$, and thus $\Delta$ is a $\Sigma$-map.

For each term $f\left(t_{1}, \ldots, t_{n}\right) \in \mathbf{T}_{M}$, we have that $f\left(\mathcal{V}_{M}\left(t_{1}\right), \ldots, \mathcal{V}_{M}\left(t_{n}\right)\right) \approx$ $\mathcal{V}_{M}(t) \in D_{1}$, and thus $\mathcal{M}(t)=\mathcal{V}_{M}(t)$. Thus, $\mathcal{M}$ satisfies all equalities between pairs of terms in the same equivalence class of $M_{c}^{*}$. Since $M_{c}^{*}$ is $T$-satisfiable, we have that $\mathcal{M}$ satisfies all disequalities in $M_{c}^{*}$ as well. Since $M_{c}^{*}$ is a superset of $M$, we have that $\mathcal{M}$ satisfies $M$.

\section{Model-Based Quantifier Instantiation}

We now focus our attention on Step 3 of our finite model finding procedure FM-Solve $_{\mathcal{H}}$ from Figure 3. In this step, the procedure FM-Solve $\mathcal{H}_{\mathcal{H}}$ considers quantified formulas in the set:

$$
\{\forall \mathbf{x} \varphi \mid(a \Leftrightarrow \forall \mathbf{x} \varphi) \in A \text { and } a \in M\}
$$

Call this set $Q$. For each formula $\forall \mathbf{x} \varphi \in Q$, it uses a quantifier instantiation heuristic $\mathcal{H}$ that returns a set of substitutions from $\mathbf{x}$ to terms in the set $\mathbf{V}$ constructed in Step 2. A trivial way to implement $\mathcal{H}$ is to choose all such possible substitutions. If $\mathbf{x}$ is a tuple of $n$ variables each ranging of a sort with $k$ domain elements, this heuristics will return $k^{n}$ substitutions, which is clearly unfeasible unless both $k$ and $n$ are rather small. Significantly more scalable heuristics can be adopted if it is possible to identify sets of substitutions $\sigma$ yielding instances $\varphi \sigma$ that are already satisfied by the current candidate model, as these substitutions can be safely ignored. These heuristics are collectively known as model-based quantifier instantiation.

A way to perform model-based quantifier instantiation, as implemented in the SMT solver Z3 [17, is to use the SMT solver itself as an oracle: a separate copy of the SMT solver is run on another query to determine whether a candidate model $\mathcal{M}$ satisfies each quantified formula. If it does not, a single instance that is falsified by $\mathcal{M}$ is added to the current clause set $F$. This approach incurs the performance overhead of constructing the corresponding query as well as initializing the oracle. Our version of model-based instantiation relies instead upon specialized data structures when checking candidate models and choosing instantiations, and may add more than one instantiation per invocation.

We describe below a model-based quantifier instantiation method that identifies entire sets of instances as satisfiable in $\mathcal{M}$ without actually generating and checking those instances individually [30]. The main idea is to determine the satisfiability in $\mathcal{M}$ of some instance $\varphi \sigma$ of a quantified formula $\forall \mathbf{x} \varphi \in Q$, generalize $\varphi \sigma$ to a set $J$ of instances equisatisfiable with $\varphi \sigma$ in $\mathcal{M}$, and then look for further instances only outside that set. The set $J$ is computed by identifying which variables of $\varphi$ actually matter in determining the satisfiability of $\varphi \sigma$. Technically, for each $\psi=\forall \mathbf{x} \varphi$, substitution $\sigma=\{\mathbf{x} \mapsto \mathbf{v}\}$ into $\mathbf{V}$, and instance $\varphi^{\prime}=\varphi \sigma$ of $\psi$, if $\mathcal{M}=\varphi^{\prime}$ we compute a partition of $\mathbf{x}$ into $\mathbf{x}_{1}$ and $\mathbf{x}_{2}$ and a corresponding partition of $\mathbf{v}$ into $\mathbf{v}_{1}$ and $\mathbf{v}_{2}$ such that $\mathcal{M} \models \forall \mathbf{x}_{2} \varphi\left\{\mathbf{x}_{1} \mapsto \mathbf{v}_{1}\right\}$; similarly, if $\mathcal{M} \not \models \neg \varphi^{\prime}$ we compute a partition such that $\mathcal{M} \forall \forall \forall \mathbf{x}_{2} \neg \varphi\left\{\mathbf{x}_{1} \mapsto \mathbf{v}_{1}\right\}$. In either case, we then know that all instances of $\varphi\left\{\mathbf{x}_{1} \mapsto \mathbf{v}_{1}\right\}$ over $\mathbf{V}$ are equisatisfiable with $\varphi^{\prime}$ in $\mathcal{M}$, 


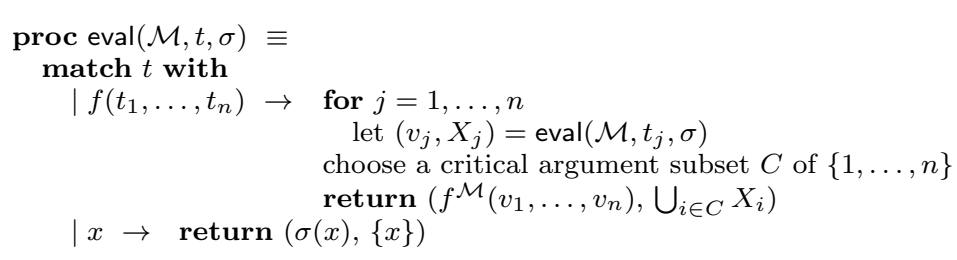

Fig. 10 The eval procedure for candidate model $\mathcal{M}$. Returns a pair $(v, S)$ where $(t \sigma)^{\mathcal{M}}=v$, and $S$ is a subset of the domain of $\sigma$ that was used to compute this interpretation.

and so it is enough to consider just $\varphi^{\prime}$ in lieu of all them. We will refer to the elements of $\mathbf{x}_{1}$ above as a set of critical variables for $\varphi$ (under $\sigma$ ) —although strictly speaking this is a misnomer as we do not insist that $\mathbf{x}_{1}$ be minimal.

\subsection{Generalizing Evaluations}

We have developed a general procedure that, given the $\Sigma$-map of a candidate model $\mathcal{M}$, a term $t$, and a substitution $\sigma$ over $t$ 's variables, computes and returns both the value of $t \sigma$ in $\mathcal{M}$ and a set of critical variables for $\sigma$. This procedure effectively extends to quantifier-free formulas as well by treating them as Boolean terms - which evaluate to either true or false in a $\Sigma$-interpretation depending on whether they are satisfied by the model or not.

The procedure, called eval, is defined recursively over its input term and is sketched in Figure 10. For uniformity, we assume that function symbols and logical operators are all in prefix form.

When evaluating a non-variable term $f\left(t_{1}, \ldots, t_{n}\right)$, eval determines a critical argument subset $C$ for it. This is a subset of $\{1, \ldots, n\}$ such that the term $f\left(s_{1}, \ldots, s_{n}\right)$ denotes a constant function in $\mathcal{M}$ where each $s_{i}$ is the value computed by eval for $t_{i}$ if $i \in C$, and is a unique variable otherwise. If $f$ is a logical symbol, the choice of $C$ is dictated by the symbol's semantics. For instance, for $\approx\left(t_{1}, t_{2}\right), C$ is $\{1,2\}$; for $\vee\left(t_{1}, \ldots, t_{n}\right)$, it is $\{1, \ldots, n\}$ if the disjunction evaluates to false; otherwise, we it chooses $\{i\}$ for some $i$ where $t_{i}$ evaluates to true. If $f$ is a function symbol of $\Sigma$, eval computes $C$ by first constructing a custom index data structure for interpreting applications of $f$ to values. The key feature of this data structure is that it uses information on the sets $X_{1}, \ldots X_{n}$ to choose an evaluation order for the arguments of $f$. For example, given the term $t=f\left(g(x, y, z), v_{2}, h(x)\right)$, say that eval computes the values $v_{1}, v_{2}, v_{3}$ and the critical variable sets $\{x, y, z\}$, $\emptyset,\{x\}$ for the three arguments of $f$, respectively. With those sets, it will use the evaluation order $(2,3,1)$ for those arguments - meaning that the second argument is evaluated first, then the third, etc. Using the index data structure, it will first determine if $f\left(x_{1}, v_{2}, x_{3}\right)$ has a constant interpretation in $\mathcal{M}$ for all $x_{1}, x_{3}$. If so, then the evaluation of $t$ depends on none of its variables, and the returned set of critical variables for $t$ will be $\emptyset$. Otherwise, if $f\left(x_{1}, v_{2}, v_{3}\right)$ has a constant interpretation in $M$, then the evaluation of $t$ depends on $\{x\}$, or else it depends on the entire variable set $\{x, y, z\}$.

The next example gives more details on the whole process of using eval to generalize a ground instance to a set of ground instances equisatisfiable with it in a given model. 
Example 8 Let $Q=\left\{\forall x_{1} x_{2} f\left(x_{1}\right) \approx g\left(x_{2}, b\right) \vee h\left(x_{2}, x_{1}\right) \not \approx b\right\}$, where all terms are of some sort S. Consider a candidate model $\mathcal{M}$ induced by a $\Sigma$-map containing the following definitions :

$$
\begin{aligned}
& \Delta_{g}=\left\{g(a, a) \approx c, g\left(y_{1}, b\right) \approx a, g\left(y_{1}, y_{2}\right) \approx b\right\} \\
& \Delta_{f}=\left\{f(b) \approx b, f\left(y_{1}\right) \approx a\right\} \\
& \Delta_{h}=\left\{h\left(y_{1}, y_{2}\right) \approx b\right\}
\end{aligned}
$$

Suppose $\mathbf{V}_{S}=\{a, b, c\}$. The table below shows the bottom-up calculation performed by eval on the formula $\varphi=f\left(x_{1}\right) \approx g\left(x_{2}, b\right) \vee h\left(x_{2}, x_{1}\right) \not \approx b$ with $\mathcal{M}$ above and $\sigma=\left\{x_{1} \mapsto a, x_{2} \mapsto a\right\}$.

\begin{tabular}{|c|l|c|}
\hline input & output & critical arg. subset \\
\hline$x_{1}$ & $\left(a,\left\{x_{1}\right\}\right)$ & \\
$x_{2}$ & $\left(a,\left\{x_{2}\right\}\right)$ & \\
$b$ & $(b, \emptyset)$ & $\emptyset$ \\
$f\left(x_{1}\right)$ & $\left(a,\left\{x_{1}\right\}\right)$ & $\{1\}$ \\
$g\left(x_{2}, b\right)$ & $(a, \emptyset)$ & $\{2\}$ \\
$h\left(x_{2}, x_{1}\right)$ & $(b, \emptyset)$ & $\emptyset$ \\
$f\left(x_{1}\right) \approx g\left(x_{2}, b\right)$ & $\left(\right.$ true,$\left.\left\{x_{1}\right\}\right)$ & $\{1,2\}$ \\
$h\left(x_{2}, x_{1}\right) \not b$ & $($ false,,$\emptyset)$ & $\{1,2\}$ \\
$f\left(x_{1}\right) \approx g\left(x_{2}, b\right) \vee h\left(x_{2}, x_{1}\right) \not b$ & $\left(\right.$ true,$\left.\left\{x_{1}\right\}\right)$ & $\{1\}$ \\
& & \\
\hline
\end{tabular}

For most entries in the table the evaluation is straightforward. For a more interesting case, consider the evaluation of $g\left(x_{2}, b\right)$. First, the arguments of $g$ are evaluated, respectively to $\left(a,\left\{x_{2}\right\}\right)$ and $(b, \emptyset)$. Using an indexing data structure built from $\Delta_{g}$ for the evaluation order $(2,1)$, we determine that $g\left(x_{2}, b\right)$ has constant value $a$ for all $x_{2}$. Hence we return an empty set of critical variables for $g\left(x_{2}, b\right)$.

Similarly, the fact that eval returns (true, $\left\{x_{1}\right\}$ ) for the original input formula $\varphi$ and the substitution $\sigma=\left\{x_{1} \mapsto a, x_{2} \mapsto a\right\}$ means that we were able to determine that all ground instances of $\varphi\left\{x_{1} \mapsto a\right\}=\left(f(a) \approx g\left(x_{2}, b\right) \vee h\left(x_{2}, a\right) \not b\right)$, not just the instance $\varphi \sigma$, are satisfied in $\mathcal{M}$. We can then use this information in FM-Solve $_{\mathcal{H}}$ to completely avoid generating and checking those instances.

\subsection{A Model-Based Instantiation Heuristic}

For any given quantified formula $\psi$, the eval procedure allows us to identify a set of instances over $\mathbf{V}$ that can be represented by a single one, as far as satisfiability in the candidate model $\mathcal{M}$ is concerned. In this subsection, we present a quantifier instantiation heuristic that generates a set $I$ of instances that together represent all instances of $\psi$ over $\mathbf{V}$ that are falsified by $\mathcal{M}$. This kind of exhaustiveness is crucial because it allows us to conclude that $\mathcal{M} \models \psi$ by just checking that $I$ is empty.

The heuristic is implemented by a procedure that relies on eval for computing the set $I$ above, or rather, a set of substitutions for generating the elements of $I$ from $\psi$. The procedure is fairly unsophisticated and quite conservative in its choice of representative instances, which makes it very simple to implement and 


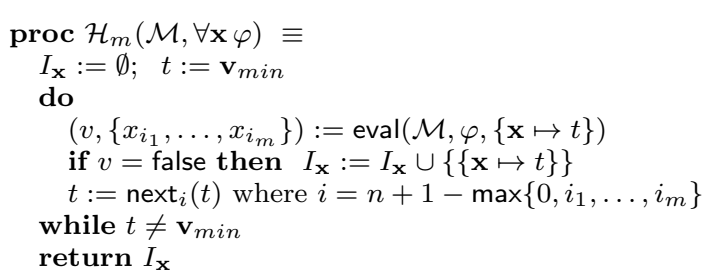

Fig. 11 A model-based instantiation heuristic $\mathcal{H}_{m}$, where $\mathbf{x}=\left(x_{1}, \ldots, x_{n}\right)$.

prove correct. Its main shortcoming is that it does not take full advantage of the information provided by eval, and so may end up producing more representative instances than needed in many cases.

Let $\psi=\forall \mathbf{x} \varphi \in Q$ with $\mathbf{x}=\left(x_{1}, \ldots, x_{n}\right)$, where $Q$ is the set defined in (2). For $i=1, \ldots, n$, let $S_{i}$ be the sort of $x_{i}$ and let $\mathbf{V}_{\mathbf{x}}=\mathbf{V}_{S_{1}} \times \cdots \times \mathbf{V}_{S_{n}}$. For each $S \in\left\{S_{1}, \ldots, S_{n}\right\}$, let $<_{S}$ be an arbitrary total ordering over the values $\mathbf{V}_{S}$ of sort $S$. Let $<$ be the lexicographic extension of these orderings to the tuples in $\mathbf{V}_{\mathbf{x}}$ and observe that $\mathbf{V}_{\mathbf{x}}$ is totally ordered by $<$. We write $\mathbf{v}_{\min }$ to denote the minimum of $\mathbf{V}_{\mathbf{x}}$ with respect to this ordering.

For every $\mathbf{v}=\left(v_{1}, \ldots, v_{n}\right) \in \mathbf{V}_{\mathbf{x}}$ Let next ${ }_{i}(\mathbf{v})$ denote the smallest tuple $\mathbf{u}$ with respect to $<$ such that $\mathbf{v}(j)<_{S_{j}} \mathbf{u}(j)$ for some $1 \leq j \leq n+1-i$, if such a tuple exists, and denote $\mathbf{v}_{\text {min }}$ otherwise (including when $\left.\left.i>n\right)\right]^{7}$ For instance, with $n=3, S_{1}=S_{2}=S_{3}$ and $\mathbf{V}_{S_{1}}=\{a, b\}$ with $a<_{S_{1}} b$, we have that next $\operatorname{ta}_{1}(a, a, a)=$ $(a, a, b), \operatorname{next}_{2}(a, a, a)=(a, b, a), \operatorname{next}_{2}(a, b, a)=(b, a, a), \operatorname{next}_{3}(a, a, a)=(b, a, a)$, and $\operatorname{next}_{2}(b, b, a)=\mathbf{v}_{\text {min }}=(a, a, a)$. Note that except in the case that next $\operatorname{nat}_{i}(\mathbf{v})$ is $\mathbf{v}_{\text {min }}$, we have that $\mathbf{v}<\operatorname{next}_{i}(\mathbf{v})$.

Our instantiation heuristic $\mathcal{H}_{m}$ is given in Figure 11. It takes in a quantifierfree formula $\varphi$ with variables $\mathbf{x}$ and returns a set $I_{\mathbf{x}}$ of substitutions $\sigma$ for $\mathbf{x}$ such that $\mathcal{M} \vDash \varphi \sigma$. At each execution of its loop the procedure implicitly determines with eval a set $I$ of instances of $\varphi$ that are equisatisfiable with $\varphi\{\mathbf{x} \mapsto \mathbf{v}\}$ in $\mathcal{M}$, where $\mathbf{v}$ is the tuple stored in the program variable $t$. The next value $t_{\text {next }}$ for $t$ is a greater tuple chosen to maintain the invariant that all the tuples between $t$ and $t_{\text {next }}$ generate instances of $\varphi$ that are in $I$. To see that, it suffices to observe that these tuples differ from $t$ only in positions that correspond to non-critical variables of $\varphi$, namely those before position $i$ where $x_{i}$ is the first critical variable of $\varphi$ in the enumeration $x_{1}, \ldots, x_{n}$. This observation is the main argument in the proof of the following result.

Lemma 3 Let $\mathbf{v}_{0}, \ldots, \mathbf{v}_{m}$ be all values successively taken by variable $t$ at the beginning of the loop in $\mathcal{H}_{m}$. Let $v_{\max }$ be the maximum element of $\mathbf{V}_{\mathbf{x}}$. Then for all $j=1, \ldots, m$,

1. $\mathbf{v}_{j-1}<\mathbf{v}_{j}$,

2. for all $\mathbf{u}$ with $\mathbf{v}_{j-1} \leq \mathbf{u}<\mathbf{v}_{j}, \mathcal{M}=\varphi\{\mathbf{x} \mapsto \mathbf{u}\}$ iff $\mathcal{M}=\varphi\left\{\mathbf{x} \mapsto \mathbf{v}_{j-1}\right\}$,

3. for all $\mathbf{u}$ with $\mathbf{v}_{m} \leq \mathbf{u} \leq \mathbf{v}_{\max }, \mathcal{M} \models \varphi\{\mathbf{x} \mapsto \mathbf{u}\} \quad$ iff $\mathcal{M} \models \varphi\left\{\mathbf{x} \mapsto \mathbf{v}_{m}\right\}$.

Proof: (Sketch) The first statement is immediate since for all $j=1 \ldots m$, we have $v_{j}=\operatorname{next}_{k}\left(\mathbf{v}_{j-1}\right)$ for some $k$ and $v_{j} \neq \mathbf{v}_{\text {min }}$. To show the second statement for a $j$, assume $\mathbf{v}_{j}=\operatorname{next}_{k}\left(\mathbf{v}_{j-1}\right)$ for some $k$. For each $\mathbf{u}$ where $\mathbf{v}_{j-1} \leq \mathbf{u}<\mathbf{v}_{j}$, we have

\footnotetext{
7 Where $\mathbf{v}(j)$ and $\mathbf{u}(j)$ are the $j$-th component of $\mathbf{v}$ and $\mathbf{u}$, respectively.
} 
that $\mathbf{u}(\ell)=\mathbf{v}_{j-1}(\ell)$ for all $\ell \geq k$. For all $\ell<k$, the eval procedure determined that the variable $x_{\ell}$ was not a critical variable for $\varphi$. Since $\mathbf{u}$ and $\mathbf{v}_{i-1}$ vary on only these variables, we have $\mathcal{M} \models \varphi\{\mathbf{x} \mapsto \mathbf{u}\}$ iff $\mathcal{M} \models \varphi\left\{\mathbf{x} \mapsto \mathbf{v}_{j-1}\right\}$. The third statement holds for similar reasons as the second.

Theorem 5 The set $I_{\mathbf{x}}$ returned by $\mathcal{H}_{m}(\mathcal{M}, \varphi, \mathbf{x})$ is empty if and only if $\mathcal{M} \models$ $\forall \mathbf{x} \varphi$.

Proof: Thanks to the previous lemma, if there is an instance of $\varphi$ that is falsified by $\mathcal{M}$, then $\mathcal{H}_{m}$ will consider at least one $\mathbf{v}_{i}$ for which $\varphi\left\{\mathbf{x} \mapsto \mathbf{v}_{i}\right\}$ evaluates to false, and hence it will return at least one instance. Conversely, if all instances of $\varphi$ are satisfied by $\mathcal{M}$, then all instances of $\varphi$ considered by $\mathcal{H}_{m}$ evaluate to true, and hence it will return no instances.

We remark that, for the model finding purposes of procedure FM-Solve $\mathcal{H}$, there is no need for the procedure $\mathcal{H}_{m}$ to compute the full set $I_{\mathbf{x}}$ once it contains at least one substitution. Any non-empty subset would suffice to trigger a (more incremental) revision of the current candidate model $\mathcal{M}$. That said, our current implementation does compute the whole set and adds all the corresponding instances to the clause set $F$ before computing another model for it. Our experiments show that computing and using one substitution at a time is worse for overall performance than computing and using the full set $I_{\mathbf{x}}$.

Example 9 Consider the quantified formula $\forall x_{1} x_{2} \varphi$ and candidate model $\mathcal{M}$ from Example 8. Assume that $a<_{S} b<_{S} c$. The result of running $\mathcal{H}_{m}$ on $\mathcal{M}, \varphi$ and $\mathbf{x}=\left(x_{1}, x_{2}\right)$ is summarized in the table below. Each row in the column shows the value of variable $t$ at the beginning of the loop in $\mathcal{H}_{m}$, the result of computing eval, the substitution (if any) added to $I_{\mathbf{x}}$ on that iteration, and the computation of the next tuple of terms next ${ }_{i}(t)$.

\begin{tabular}{|c|c|c|c|c|c|}
\hline Iteration & $t$ & eval $(\mathcal{M}, \varphi,\{\mathbf{x} \mapsto t\})$ & Add to $I_{\mathbf{x}}$ & $i$ & $\operatorname{next}_{i}(t)$ \\
\hline 1 & $(a, a)$ & (true,$\left.\left\{x_{1}\right\}\right)$ & $\emptyset$ & 2 & $(b, a)$ \\
2 & $(b, a)$ & (false, $\left.\left\{x_{1}\right\}\right)$ & $\left\{x_{1} \mapsto b, x_{2} \mapsto a\right\}$ & 2 & $(c, a)$ \\
3 & $(c, a)$ & (true,$\left.\left\{x_{1}\right\}\right)$ & $\emptyset$ & 2 & $(a, a)$ \\
\hline
\end{tabular}

We begin by setting $t$ to $\mathbf{v}_{\text {min }}=(a, a)$. As demonstrated in Example 8, we have that $\operatorname{eval}\left(\mathcal{M}, \varphi,\left\{x_{1} \mapsto a, x_{2} \mapsto a\right\}\right)$ returns the pair (true, $\left.\left\{x_{1}\right\}\right)$. The first component of this pair indicates that $\mathcal{M} \llbracket \varphi\left\{x_{1} \mapsto a, x_{2} \mapsto a\right\} \rrbracket=$ true, and hence we do not add this substitution to $I_{\mathbf{x}}$. The second component of this pair indicates moreover that this interpretation did not depend on the value of $x_{2}$, and hence $\left(\varphi\left\{x_{1} \mapsto a, x_{2} \mapsto v\right\}\right)^{\mathcal{M}}=$ true for all values of $v$. Thus, we need not consider $t=(a, b)$ or $t=(a, c)$. Instead, on the second iteration, we consider next $_{2}(a, a)=(b, a)$. Subsequently, eval $\left(\mathcal{M}, \varphi,\left\{x_{1} \mapsto b, x_{2} \mapsto a\right\}\right)$ returns the pair (false, $\left.\left\{x_{1}\right\}\right)$. This indicates that $\left(\varphi\left\{x_{1} \mapsto b, x_{2} \mapsto v\right\}\right)^{\mathcal{M}}=$ false for all values of $v$. We add the substitution $\left\{x_{1} \mapsto b, x_{2} \mapsto a\right\}$ to $I_{\mathbf{x}}$ only. Finally, on the third iteration, $\operatorname{eval}\left(\mathcal{M}, \varphi,\left\{x_{1} \mapsto c, x_{2} \mapsto a\right\}\right)$ returns the pair (true, $\left\{x_{1}\right\}$ ); we add no substitutions to $I_{\mathbf{x}}$, and the loop terminates. Overall, $\mathcal{H}_{m}$ returns the singleton set of substitutions $\left\{\left\{x_{1} \mapsto b, x_{2} \mapsto a\right\}\right\}$.

\subsection{Enhancement: Heuristic Instantiation}

Modern SMT solvers rely on syntatic heuristic instantiation methods for finding unsatisfiable instances for quantified formulas [13, 25, 31. In these methods, quan- 
tified formulas are instantiated based on pattern matching. For instance, the solver may choose to instantiate the quantified formula $\forall x P(f(x)) \Rightarrow Q(x)$ based on the substitution $\{x \mapsto c\}$ when $P(f(c))$ is a ground term occurring in its current satisfying assignment. This technique is often referred to as E-matching. We found that heuristic instantiation-based E-matching can be helpful in the context of our finite model finding approach as well, because the instances it generates are helpful in quickly ruling out candidate models that are obviously spurious.

A quantifier instantiation heuristic $\mathcal{H}$, such as the model-based one from the previous section, can be enhanced by applying heuristic instantiation with a higher priority. That is, we may consider a modified quantifier instantiation heuristic that first computes the set of instances $I_{\mathbf{x}}$ returned by E-matching for a quantified formula $\forall \mathbf{x} \varphi$. If this set is non-empty, it returns $I_{\mathbf{x}}$; otherwise it returns the instances from the original heuristic $\mathcal{H}$ on $\forall \mathbf{x} \varphi$.

In practice, we have found that it is best to apply heuristic quantifier instantiation after finding a satisfying assignment with a bounded number of equivalence classes. By waiting to apply quantifier instantiation until after a satisfying assignment of this form can be constructed, we can avoid pitfalls common to $E$ matching. In particular, having a finite cardinality for uninterpreted sorts ensures that only a finite number of terms are unique up to congruence, thus ensuring that $E$-matching, which is non-terminating in general, will eventually return instances that rule out the current upper bound on cardinality, or terminate with no instances. We discuss the impact of heuristic instantiation further in Section 9.2

\section{Results}

We implemented all features mentioned in this paper inside CVC4 2, a stateof-the-art SMT solver based on the $\operatorname{DPLL}\left(T_{1}, \ldots, T_{m}\right)$ architecture. This section presents experimental results on this implementation 8 We separate this section into two sets of experiments, the first to evaluate the relative effectiveness of various strategies for the FCC solver, and the second to evaluate the model finder's overall performance when used with quantified formulas. For the second set of experiments, we compare our model finder against state-of-the-art SMT solvers and automated theorem provers.

\subsection{FCC Solver Evaluation}

We first examine the effectiveness of approach to handling ground problems in the theory of EUF with finite cardinality constraints (FCC). In this section, all experiments were run on a Linux machine with an 8 -core $2.60 \mathrm{GHz}$ Intel ${ }^{\circledR}$ Xeon ${ }^{\circledR}$ E5-2670 processor with 16GB of RAM.

We tested various configurations of the FCC solver, starting with the default configuration cvc4+f, which contains the region-based enhancements described in Section 6.2, where conflicting states are reported by using clique lemmas of the form ( $\neg$ distinct $\left.\left(t_{1}, \ldots, t_{k+1}\right) \vee \neg \operatorname{card}_{S, k}\right)$. We also tested a configuration, cvc4+fe, which reports conflict clauses of the form $\left(\bar{l}_{1} \vee \ldots \vee \bar{l}_{n} \vee \neg \operatorname{card}_{S, k}\right)$, where $l_{1}, \ldots, l_{n}$

\footnotetext{
8 Details can be found at http://cs.uiowa.edu/ ajreynol/TPLP-fmf
} 

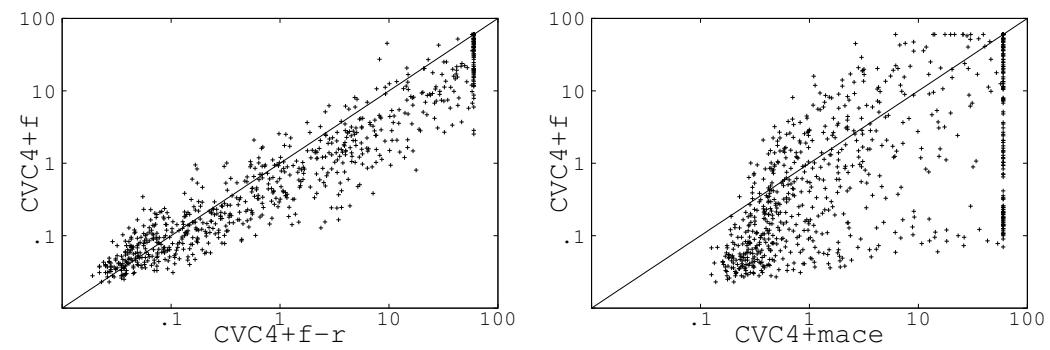

Fig. 12 Results for randomly generated benchmarks. Runtimes are on a log-log scale.

are equalities and disequalities that entail distinct $\left(t_{1}, \ldots, t_{k+1}\right)$. This configuration avoids the introduction of new equalities into the search (contained in the expansion of distinct), but has the disadvantage that it can generate different conflict clauses for essentially the same clique. Additionally, we considered configuration $\mathbf{c v c 4}+\mathbf{f}-\mathbf{r}$, which differs from $\mathbf{c v c 4}+\mathbf{f}$ only in that regionalizations have always just one region per sort $S$, encompassing the entire disequality graph for $S$.

We also evaluated the MACE-style approach to finite model finding described in related work 24, which we implemented in the configuration cvc4+mace. In the case of a set of ground clauses $F$ involving a single sort, if $\mathbf{T}_{F}$ is the set of all terms in $F$ and $c_{1}, \ldots, c_{k}$ are fresh constants serving as domain constants, this configuration checks the satisfiability of

$$
F \wedge \operatorname{distinct}\left(c_{1}, \ldots, c_{k}\right) \wedge \bigwedge_{t \in \mathbf{T}_{F}}\left(t \approx c_{1} \vee \ldots \vee t \approx c_{k}\right)
$$

for $k=1,2, \ldots$ until $(3)$ is found satisfiable for some $k$. Then, the minimal model size for $F$ is $k$. A major and well-known shortcoming of this approach is the introduction of unwanted symmetries in the problem due to the use of domain constants. CVC4 can address this issue to some extent since it incorporates symmetry breaking techniques directly at the ground EUF level [12.

We considered satisfiable benchmarks encoding randomly generated graph coloring problems and consisting of a conjunction of disequalities between constants of a single sort. In particular, we considered a total of 793 non-trivial problems containing between 20 and 50 unique constants and between 100 and 900 disequalities, and measured the time it takes each configuration to find a model of minimum size, with a 60 second timeout. For the benchmarks we tested, the configuration $\mathbf{c v c} 4+\mathbf{f}$ solves the most benchmarks within the time limit: 723 . The configuration $\mathbf{c v c 4}+\mathbf{f}$ was an order of magnitude faster than cvc4+fe on most benchmarks, with the latter only being able to solve 309 benchmarks within the time limit. This strongly suggests that generating explanations for cliques in conflict lemmas involving cardinality constraints is not an effective approach in this scheme.

Figure 12 compares the performance of the configuration $\mathbf{c v c} \mathbf{4}+\mathbf{f}$ against $\mathbf{c v c} 4+\mathbf{f}-\mathbf{r}$ and $\mathbf{c v c} 4+$ mace. The second scatter plot clearly shows that the $\mathbf{c v c} \mathbf{4}+\mathbf{f}$ configuration generally requires less time and solves more benchmarks (723 vs. 664) than cvc4+f-r, confirming the usefulness of a region-based approach for clique detection. The third scatter plot compares cvc4+f against cvc4+mace. The latter configuration was able to solve only 617 benchmarks and generally 
performed poorly on benchmarks with larger model size. The median model size of the 123 benchmarks solved only by $\mathbf{c v c} 4+\mathbf{f}$ was 17 , whereas the median size of the 13 benchmarks solved only by cvc4+mace was 10 . This suggests that for larger cardinalities cvc4+mace suffers from the model symmetries created by the introduction of domain constants, something that $\mathbf{c v c} 4+\mathbf{f}$ avoids.

\subsection{Finite Model Finder Evaluation}

We provide results on CVC4 with finite model finding for three sets of benchmarks coming from different formal methods applications, including verification and automated theorem proving. We will refer to various configurations of CVC4 based on the features they include. Configuration $\mathbf{c v c} 4+\mathbf{f}$ uses the finite model finding techniques described earlier. Additionally, configurations containing $\mathbf{m}$ in their suffix use the model-based quantifier instantiation heuristic described in Section 8, and configurations with $\mathbf{i}$ use heuristic instantiation, which can be paired with finite model finding configurations as described in Section 8.3

Experiments from Section ?? were run on a Linux machine with an 8-core 2.60GHz Intel ${ }^{\circledR}$ Xeon ${ }^{\circledR}$ E5-2670 processor. All others were run on a Linux machine with an 8 -core $3.20 \mathrm{GHz}$ Intel ${ }^{\circledR}$ Xeon ${ }^{\circledR}$ E5-1650 processor with $16 \mathrm{~GB}$ of RAM.

\section{Intel benchmarks}

We evaluated the overall effectiveness of CVC4's finite model finder for quantified SMT formulas taken from verification conditions generated by DVF [18], a tool used at Intel for verifying properties of security protocols and design architectures, among other applications. Both unsatisfiable and satisfiable benchmarks were produced, the latter by manually removing necessary assumptions from verification conditions. All benchmarks contain quantifiers, although only over uninterpreted sorts, and span a wide range of theories, including linear integer arithmetic, arrays, EUF, and algebraic datatypes.

For comparison we looked at the SMT solvers CVC3 [4] (version 2.4.1) $)^{9}$. Yices [14 (version 1.0.32), and z3 [1] (version 4.1). We did not consider traditional theorem provers and finite model finders because they do not have built-in support for the theories in our benchmark set. All these solvers use E-matching as a heuristic method for answering unsatisfiable in the presence of universally quantified formulas. Z3 additionally relies on model-based quantifier instantiation techniques to establish satisfiability in the presence of quantified formulas [17.

The results, separated into unsatisfiable and satisfiable instances, are shown in Figure 13 for five classes of benchmarks and a timeout of 600 seconds per benchmark. The first two classes, refcount and german, represent verification conditions for systems described in [18; benchmarks in the third are taken from [37]; the last two classes are verification problems internal to Intel.

For the satisfiable benchmarks, our finite model finder is the only tool capable of solving any instance in the last three benchmark classes. In fact, $\mathbf{c v c} 4+\mathbf{f}$

\footnotetext{
9 CVC3 is the predecessor of CVC4. The latter was developed from scratch, and does not have any code in common with CVC3.
} 


\begin{tabular}{|l|c|c|c|c|c|r|r|r|r|r|}
\hline Sat & \multicolumn{2}{|c|}{$\begin{array}{c}\text { german } \\
(45)\end{array}$} & \multicolumn{2}{c|}{$\begin{array}{c}\text { refcount } \\
(6)\end{array}$} & \multicolumn{2}{c|}{$\begin{array}{c}\text { agree } \\
(42)\end{array}$} & \multicolumn{2}{|c|}{$\begin{array}{c}\text { apg } \\
(19)\end{array}$} & \multicolumn{2}{c|}{$\begin{array}{l}\text { bmk } \\
(37)\end{array}$} \\
\hline & $\#$ & time & $\#$ & time & $\#$ & time & $\#$ & time & $\#$ & time \\
\hline cvc3 & 0 & 0.0 & 0 & 0.0 & 0 & 0.0 & 0 & 0.0 & 0 & 0.0 \\
yices & 2 & 0.0 & 0 & 0.0 & 0 & 0.0 & 0 & 0.0 & 0 & 0.0 \\
z3 & 45 & 1.1 & 1 & 7.0 & 0 & 0.0 & 0 & 0.0 & 0 & 0.0 \\
cvc4+i & 2 & 0.0 & 0 & 0.0 & 0 & 0.0 & 0 & 0.0 & 0 & 0.0 \\
cvc4+f & $\mathbf{4 5}$ & 0.3 & $\mathbf{6}$ & 0.1 & 42 & 15.5 & 18 & 200.0 & 36 & 1201.5 \\
cvc4+f-r & $\mathbf{4 5}$ & 0.3 & $\mathbf{6}$ & 0.1 & 42 & 18.6 & 15 & 364.3 & 34 & 720.4 \\
cvc4+fi & 45 & 0.4 & $\mathbf{6}$ & 0.1 & $\mathbf{4 2}$ & 14.2 & 19 & 492.8 & 36 & 831.0 \\
cvc4+fm & $\mathbf{4 5}$ & 0.3 & $\mathbf{6}$ & 0.1 & 42 & 23.6 & $\mathbf{1 9}$ & 210.2 & 37 & 375.1 \\
cvc4+fmi & $\mathbf{4 5}$ & 0.3 & $\mathbf{6}$ & 0.1 & 42 & 16.4 & 19 & 221.1 & $\mathbf{3 7}$ & 176.8 \\
\hline
\end{tabular}

\begin{tabular}{|c|c|c|c|c|c|c|c|c|c|c|}
\hline \multirow[t]{2}{*}{ Unsat } & \multicolumn{2}{|c|}{$\begin{array}{c}\underset{(145)}{\text { german }} \\
\text { (145) }\end{array}$} & \multicolumn{2}{|c|}{$\begin{array}{c}\text { refcount } \\
(40)\end{array}$} & \multicolumn{2}{|c|}{$\begin{array}{c}\text { agree } \\
(488)\end{array}$} & \multicolumn{2}{|c|}{$\begin{array}{l}\text { apg } \\
(304)\end{array}$} & \multicolumn{2}{|c|}{$\begin{array}{l}\text { bmk } \\
(244)\end{array}$} \\
\hline & \# & time & \# & time & \# & time & \# & time & \# & time \\
\hline cve3 & 145 & 0.4 & 40 & 0.2 & 457 & 6.8 & 267 & 77.0 & 229 & 76.2 \\
\hline yices & 145 & 1.8 & 40 & 7.0 & 488 & 1475.4 & 304 & 35.8 & 244 & 25.3 \\
\hline z3 & 145 & 1.9 & 40 & 0.9 & 488 & 10.6 & 304 & 12.2 & 244 & 5.3 \\
\hline $\operatorname{cvc} 4+\mathrm{i}$ & 145 & 0.1 & 40 & 0.2 & 484 & 6.8 & 304 & 11.2 & 244 & 2.9 \\
\hline $\operatorname{cvc} 4+f$ & 145 & 0.8 & 40 & 0.4 & 476 & 3782.1 & 298 & 2252.5 & 242 & 1507.0 \\
\hline $\operatorname{cvc} 4+f-r$ & 145 & 0.4 & 40 & 0.2 & 475 & 1574.3 & 294 & 3836.0 & 240 & 1930.5 \\
\hline $\operatorname{cvc} 4+f i$ & 145 & 0.7 & 40 & 0.1 & 488 & 188.7 & 302 & 342.0 & 244 & 660.3 \\
\hline $\operatorname{cvc} 4+\mathrm{fm}$ & 145 & 0.4 & 40 & 0.3 & 471 & 5018.2 & 300 & 1122.7 & 242 & 834.1 \\
\hline cve $4+$ fmi & 145 & 0.3 & 40 & 0.1 & 488 & 185.9 & 302 & 339.8 & 244 & 668.5 \\
\hline
\end{tabular}

Fig. 13 Number of solved satisfiable and unsatisfiable Intel (DVF) benchmarks and cumulative time for solved benchmarks. All times are in seconds.

is able to solve all but two, and most of them in less than a second. When extended to include techniques for model-based quantifier instantiation (configurations $\mathbf{c v c 4}+\mathbf{f m}$ and $\mathbf{c v c 4}+\mathbf{f m i}$ ), we are able to solve all satisfiable benchmarks within the timeout. By comparing $\mathbf{c v c 4}+\mathbf{f}$ against $\mathbf{c v c 4}+\mathbf{f}-\mathbf{r}$, we see that the region-based approach for recognizing cliques is beneficial, particularly for the harder classes where the latter configuration solves fewer benchmarks within the timeout. The model sizes found for these benchmarks were relatively small; only a handful had a model with sort cardinalities larger than 4. To our knowledge, our model finder is the only tool capable of solving these benchmarks.

For the unsatisfiable benchmarks, Yices and Z3 can solve all of them, with Z3 being much faster in some cases. We observe that CVC4 with finite model finding is orders of magnitude slower than the SMT solvers on these benchmarks. This is, however, to be expected since it is geared towards finding models, and applies exhaustive instantiation with increasingly large cardinality bounds, which normally delays the discovery that the problem is unsatisfiable regardless of those bounds.

However, we found that each unsatisfiable problem can be solved by either cvc4 or cvc4+fmi, and in less than 3s. Additionally, configuration cvc4+fmi solves all unsatisfiable benchmarks within 900s, suggesting that CVC4's model finder makes consistent progress towards answering unsatisfiable on provable DVF verification conditions. From the perspective of verification tools, the results here seem promising. A common strategy for handling a verification condition would be to first use an SMT solver hoping that it can quickly find it unsatisfiable with $E$-matching techniques; and then resort to finite model finding if needed to either answer unsatisfiable, or produce a model representing a concrete counterexample for the verification condition. 


\begin{tabular}{|l|c|c|c|c|c|c|c|c|c|c|}
\hline & \multicolumn{9}{|c|}{ Unsat } \\
\hline & $\begin{array}{c}\text { EPR } \\
(920)\end{array}$ & $\begin{array}{c}\text { NEQ } \\
(2008)\end{array}$ & $\begin{array}{c}\text { SEQ } \\
(7682)\end{array}$ & $\begin{array}{c}\text { PEQ } \\
(1796)\end{array}$ & $\begin{array}{c}\text { Total } \\
(12406)\end{array}$ & $\begin{array}{c}\text { EPR } \\
(388)\end{array}$ & $\begin{array}{c}\text { NEQ } \\
(618)\end{array}$ & $\begin{array}{c}\text { SEQ } \\
(340)\end{array}$ & $\begin{array}{c}\text { PEQ } \\
(612)\end{array}$ & $\begin{array}{c}\text { Total } \\
(1958)\end{array}$ \\
\hline $\mathbf{z 3}$ & 840 & 1406 & $\mathbf{3 3 6 6}$ & 656 & 6268 & 345 & 261 & 175 & 160 & 941 \\
cvc3 & 596 & 910 & 3091 & 648 & 5245 & 24 & 0 & 8 & 0 & 32 \\
iprover & $\mathbf{8 8 8}$ & $\mathbf{1 7 8 6}$ & 3346 & 310 & $\mathbf{6 3 3 0}$ & $\mathbf{3 8 4}$ & 434 & 106 & 156 & 1080 \\
iprover+f & - & - & - & - & - & 378 & $\mathbf{5 5 5}$ & $\mathbf{2 2 4}$ & 268 & 1425 \\
paradox & - & - & - & - & - & 343 & 534 & 201 & $\mathbf{3 7 2}$ & $\mathbf{1 4 5 0}$ \\
cvc4+i & 809 & 1346 & 3277 & $\mathbf{6 6 8}$ & 6100 & 21 & 1 & 8 & 0 & 30 \\
cvc4+f & 736 & 900 & 1261 & 531 & 3428 & 329 & 441 & 178 & 242 & 1190 \\
cvc4+fm & 725 & 942 & 1315 & 419 & 3401 & 329 & 448 & 214 & 286 & 1277 \\
cv44+fi & 733 & 994 & 1594 & 457 & 3778 & 329 & 422 & 178 & 231 & 1160 \\
cvc4+fmi & 748 & 997 & 1594 & 459 & 3798 & 327 & 416 & 190 & 232 & 1165 \\
\hline
\end{tabular}

Fig. 14 Number of solved TPTP benchmarks. All experiments were run with a 300 second timeout.

\section{TPTP benchmarks}

We considered benchmarks from a recent version of the TPTP library [34] (5.4.0), a widely-used library from the automated theorem proving community. The benchmarks from this library involve no theory reasoning other than equality, and are composed mostly of quantified formulas.

We compared CVC4 (version 1.2) against other SMT solvers including Z3 (version 4.3) and CVC3 (version 2.4.1), as well as various automated theorem provers and model finders for first order logic, including Paradox [10] and iProver 21] (version 0.99). Paradox is a MACE-style model finder that uses preprocessing optimizations such as sort inference and clause splitting, among others, and then encodes to SAT the original problem together with increasingly looser constraints on the size of the model. iProver is an automated theorem prover based in the Inst-Gen calculus that can also run in finite model finding mode (iprover $+\mathbf{f}$ ). In that mode, it incrementally bounds model sizes in a manner similar to MACE-style model finding. However, it encodes the whole problem into the EPR fragment 10 for which it is a decision procedure. Since these two tools are limited to classical first-order logic with equality, we considered only the unsorted first-order benchmarks of TPTP.

Figure 14 shows results for benchmarks from the TPTP library that are known to be satisfiable or unsatisfiable. All experiments were run with a 300 second timeout per benchmark. The benchmarks were placed into (exactly one) category based on its logical and syntactic characteristics, where EPR includes benchmarks that reside in the effectively propositional fragment, NEQ are benchmarks that do not contain any equality reasoning, SEQ are benchmarks containing some equality, and PEQ are benchmarks containing only pure equality. Both configurations iprover and iprover $+\mathbf{f}$ used scheduling strategies that iProver incorporated for CASC 24, a competition for automated theorem provers, meaning that multiple configurations of this solver were run sequentially. The latter of these configurations, as well as the configuration paradox were solely run on the satisfiable benchmarks from this set. All configurations of $\mathrm{CVC} 4$ with finite model finding used sort inference techniques as described in 32, which is capable of treating unsorted inputs as multi-sorted based on their structure. Sort inference techniques are known to be useful for this set of benchmarks, and are used in most competitive ATP systems, including Paradox and iProver.

10 This fragment of first-order logic consists of all formulas of the form $\exists \mathbf{x} \cdot \forall \mathbf{y} \cdot \varphi$, where $\varphi$ is quantifier-free and contains no function symbols. 

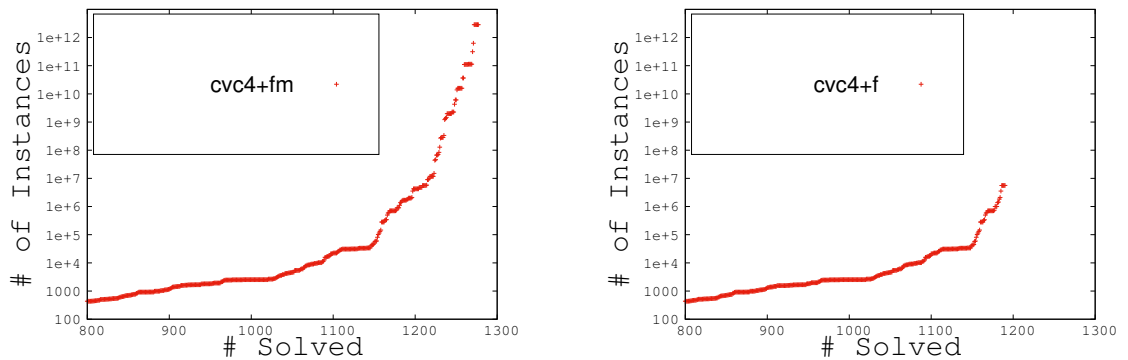

Fig. 15 Satisfiable TPTP problems with $(\mathbf{c v c 4 + f m})$ and without $(\mathbf{c v c 4}+\mathbf{f})$ model-based instantiation. A point $(x, y)$ on this graph says the configuration solves $x$ benchmarks each having at most $y$ ground instances of quantified formulas.

For satisfiable benchmarks, CVC4's model finder with exhaustive instantiation $(\mathbf{c v c 4 + f})$ solves 1190 benchmarks. Using model-based quantifier instantiation, that number goes up to 1277 (configuration cvc4+fm). Using heuristic instantiation $(\mathbf{c v c} \mathbf{4}+\mathbf{f m i})$ in addition to model-based instantiation led to finding fewer satisfiable benchmarks, solving 1165 within the timeout, suggesting that the solver becomes overloaded with the large number of instantiations produced by exhaustive instantiation.

While CVC4 solves more than Z3, which finds 941 satisfiable benchmarks, our model finder still trails the overall performance of the other model finders on these problems. Paradox was the overall best solver, finding 1450 satisfiable benchmarks. We attribute this to the fact that we have not implemented advanced preprocessing techniques, such as clause splitting, that have been shown to be critical for finding finite models of TPTP benchmarks. Nevertheless, CVC4's model finder solves more satisfiable benchmarks (214) than Paradox for classes of problems having some equality reasoning (SEQ). Collectively, some configuration of $\mathrm{CVC} 4$ with finite model finding was able to solve 52 satisfiable benchmarks that paradox was not able to solve, and 36 satisfiable benchmarks that iprover $+\mathbf{f}$ was not able to solve.

Figure 14 also shows results for unsatisfiable problems. Although these results are not comparable to those achieved by state-of-the-art theorem provers, such as Vampire and E 22,33], we note that iprover solves the most benchmarks, 6330. Here, cvc4+fmi was the best configuration of CVC4 with finite model finding, solving 3781 within the timeout. While finite model finding configurations solved considerably fewer than using heuristic instantiation alone, some configuration of $\mathrm{CVC} 4$ with finite model finding solves 144 unsatisfiable benchmarks that were unable to be solved by any other solver in these experiments, including iProver and $\mathrm{z} 3$.

To further evaluate the impact of model-based quantifier instantiation on our model finder, we recorded statistics on the domain size of quantified formulas in benchmarks solved by its various configurations. We measured the total number of possible ground instances for all quantified formulas in the smallest model for that benchmark (a quantified formula over $n$ variables each with domain size $k$ has $k^{n}$ instances). For a problem with $d$ total instances, the configuration $\mathbf{c v c} 4+\mathbf{f}$ 


\begin{tabular}{|c|c|c|c|c|c|c|c|c|c|c|}
\hline Sat & Arr & FFT & FTA & Hoare & NSS & QEp & SN & TSq & TSf & Total \\
\hline z3 & 2 & 19 & 24 & 47 & 7 & 47 & 1 & 17 & 8 & 172 \\
\hline cvc3 & 0 & 9 & 0 & 0 & 0 & 0 & 0 & 8 & 0 & 17 \\
\hline $\operatorname{cvc} 4+\mathrm{i}$ & 0 & 9 & 0 & 0 & 0 & 0 & 0 & 8 & 0 & 17 \\
\hline $\operatorname{cvc} 4+f$ & 35 & 145 & 177 & 162 & 56 & 85 & 12 & 57 & 90 & 819 \\
\hline $\operatorname{cvc} 4+\mathrm{fm}$ & 33 & 141 & 173 & 155 & 43 & 86 & 12 & 54 & 89 & 786 \\
\hline $\operatorname{cvc} 4+\mathrm{fi}$ & 36 & 146 & 172 & 162 & 61 & 86 & 12 & 55 & 93 & 823 \\
\hline $\operatorname{cvc} 4+\mathrm{fmi}$ & 36 & 147 & 174 & 162 & 61 & 83 & 12 & 56 & 93 & 824 \\
\hline Unsat & Arr & FFT & FTA & Hoare & NSS & QEp & $\overline{\text { SN }}$ & TSq & TSf & Total \\
\hline z3 & 178 & 277 & 917 & 549 & 108 & 325 & 241 & 620 & 291 & 3506 \\
\hline cvc3 & 321 & 296 & 1124 & 607 & 105 & 297 & 207 & 643 & 227 & 3827 \\
\hline $\operatorname{cvc} 4+\mathrm{i}$ & 307 & 288 & 990 & 563 & 117 & 360 & 242 & 708 & 283 & 3858 \\
\hline $\operatorname{cvc} 4+\mathrm{f}$ & 165 & 106 & 451 & 239 & 44 & 131 & 88 & 442 & 151 & 1817 \\
\hline $\operatorname{cvc} 4+\mathrm{fm}$ & 132 & 92 & 442 & 238 & 26 & 160 & 88 & 430 & 128 & 1736 \\
\hline $\operatorname{cvc} 4+\mathrm{fi}$ & 172 & 185 & 589 & 383 & 47 & 222 & 112 & 585 & 196 & 2491 \\
\hline $\operatorname{cvc} 4+\mathrm{fmi}$ & 168 & 186 & 589 & 379 & 47 & 222 & 112 & 584 & 196 & 2483 \\
\hline
\end{tabular}

Fig. 16 Number of solved satisfiable and unsatisfiable Isabelle benchmarks for various classes within a 300 second timeout.

must explicitly generate these $d$ instances, while a model-based configuration may avoid doing so.

The graph on the right hand side of Figure 15 shows that $\mathbf{c v c 4}+\mathbf{f}$ was only able to solve 13 problems having more than $100 \mathrm{~K}$ instances, the maximum having around 5.6 million instances. On the other hand, cvc4+fm was capable of solving 123 problems having more than $100 \mathrm{~K}$ instances, with the largest having more than 2.8 trillion instances. This indicates that the model-based instantiation approach improves the scalability of our model finder, and allows it to solve benchmarks where exhaustive instantiation is clearly infeasible. Model finders such as Paradox have other ways of handling the explosion in the number of instances, namely by minimizing the number of variables per clause. Coupling these techniques with model-based techniques could then lead to additional improvements in scalability. Since techniques for reducing variables in clauses rely on introducing new symbols into the problem, we have found that they have a negative impact on performance for several classes of benchmarks, and thus are disabled by default in CVC4.

\section{Isabelle benchmarks}

Recent work has shown that SMT solvers are effective at discharging proof obligations for Isabelle, a generic proof assistant [27. The performance of these solvers can benefit from an encoding that makes use of theories [7. We considered a set of 13,041 benchmarks corresponding to both provable and unprovable proof goals, corresponding to a superset of those discussed in [7. Most benchmarks in this set contain quantifiers, and a significant portion contain integer arithmetic. For many of them, the quantification is limited to the uninterpreted sorts, thus making our finite model finding approach applicable.

The results are shown in Figure 16. For satisfiable benchmarks, all configurations of CVC4's model finder find more satisfiable problems than Z3, which finds only 172 of them overall. The model-based quantifier instantiation technique from Section 8 (configuration $\mathbf{c v c 4}+\mathbf{f m}$ ) was less effective than naive instantiation (configuration cvc4+f) which solves 819 , suggesting that model-based techniques were not effective at minimizing the number of instantiations for this set of benchmarks. Using heuristic E-matching noticeably improved the search for models, 
as configuration cvc4+fi solves 823 satisfiable benchmarks. Using both modelbased instantiation and heuristic instantiation, configuration cvc4+fmi, found more satisfiable problems (824) than any other configuration.

For unsatisfiable problems, $\mathbf{c v c 4 + i}$ is the overall winner, solving 3,858 , which was more than both $\mathbf{z 3}$ and cvc3 which solved 3,506 and 3,827 respectively. Configurations of $\mathrm{CVC} 4$ with finite model finding generally solves less unsatisfiable benchmarks, but is orthogonal to other solvers and configurations. In these experiments, 309 unsatisfiable benchmarks that CVC3 cannot solve are solved by at least one configuration of CVC4 with finite model finding. Similarly, a configuration of CVC4 with finite model finding solves 429 unsatisfiable benchmarks that Z3 cannot, and 168 that $\mathbf{c v c} 4+\mathbf{i}$ cannot.

\section{Conclusion}

We developed a general approach for finite model finding in SMT that is efficient for many classes of problems that are of practical interest to formal methods applications. Experimental evidence from an implementation of these methods in the SMT solver CVC4 shows that our approach is effective in practice at solving many classes of benchmarks, including verification conditions from industry, and benchmarks from automated theorem proving libraries. The implementation is highly competitive with respect to other SMT solvers and to automated theorem provers.

In ongoing work, we plan to extend our approach to the problem of finding models of formulas with quantifiers ranging over built-in domains such as the integers and inductive datatypes. We are also investigating the use of $\mathrm{CVC} 4$ as a backend to interactive proof assistants such as Isabelle and Coq, where small counterexamples to conjectures are often helpful to the user.

Acknowledgements We would like to thank Amit Goel and Sava Krstić for their valuable contributions to this work, Sascha Böhme for proving the Isabelle benchmarks, and François Bobot for his help in writing a TPTP front end for CVC4.

\section{References}

1. Baader, F., Nipkow, T.: Term Rewriting and All That. Cambridge University Press (1998)

2. Barrett, C., Conway, C., Deters, M., Hadarean, L., Jovanovic, D., King, T., Reynolds, A., Tinelli, C.: CVC4. In: Proceedings of CAV'11, LNCS, vol. 6806, pp. 171-177. Springer (2011)

3. Barrett, C., Nieuwenhuis, R., Oliveras, A., Tinelli, C.: Splitting on demand in SAT modulo theories. In: Proceedings of LPAR'06, LNCS, vol. 4246, pp. 512-526. Springer (2006)

4. Barrett, C., Tinelli, C.: CVC3. In: W. Damm, H. Hermanns (eds.) Proceedings of the $19^{\text {th }}$ International Conference on Computer Aided Verification (CAV '07), Lecture Notes in Computer Science, vol. 4590, pp. 298-302. Springer-Verlag (2007). Berlin, Germany

5. Baumgartner, P., Bax, J., Waldmann, U.: Finite quantification in hierarchic theorem proving. In: Automated Reasoning - 7th International Joint Conference, IJCAR 2014, Held as Part of the Vienna Summer of Logic, VSL 2014, Vienna, Austria, July 19-22, 2014. Proceedings, pp. 152-167 (2014)

6. Baumgartner, P., Fuchs, A., de Nivelle, H., Tinelli, C.: Computing finite models by reduction to function-free clause logic. J. Applied Logic 7(1), 58-74 (2009) 
7. Blanchette, J.C., Böhme, S., Paulson, L.C.: Extending Sledgehammer with SMT solvers. In: N. Børner, V. Sofronie-Stokkermans (eds.) Automated Deduction, Lecture Notes in Computer Science, vol. 6803, pp. 116-130. Springer (2011)

8. Blanchette, J.C., Nipkow, T.: Nitpick: A counterexample generator for higher-order logic based on a relational model finder. In: M. Kaufmann, L.C. Paulson (eds.) ITP 2010, LNCS, vol. 6172, pp. 131-146. Springer (2010)

9. Bruttomesso, R., Cimatti, A., Franzén, A., Griggio, A., Sebastiani, R.: Delayed theory combination vs. Nelson-Oppen for satisfiability modulo theories: a comparative analysis. AMAI 55(1-2), 63-99 (2009)

10. Claessen, K., Sörensson, N.: New techniques that improve MACE-style finite model building. In: CADE-19 Workshop: Model Computation - Principles, Algorithms, Applications, pp. 11-27 (2003)

11. De Moura, L., Bjørner, N.: Z3: an efficient SMT solver. In: Proceedings of the Theory and practice of software, 14th international conference on Tools and algorithms for the construction and analysis of systems, TACAS'08/ETAPS'08, pp. 337-340. Springer-Verlag, Berlin, Heidelberg (2008)

12. Déharbe, D., Fontaine, P., Merz, S., Paleo, B.W.: Exploiting symmetry in SMT problems. In: Proceedings of CADE-23, LNCS, vol. 6803, pp. 222-236. Springer (2011)

13. Detlefs, D., Nelson, G., Saxe, J.B.: Simplify: A theorem prover for program checking. Tech. rep., J. ACM (2003)

14. Dutertre, B., De Moura, L.: The yices smt solver. Tool paper at http://yices. csl. sri. com/tool-paper. pdf 2, 2 (2006)

15. Garey, M.R., Johnson, D.S., Stockmeyer, L.: Some simplified np-complete problems. In: Proceedings of the Sixth Annual ACM Symposium on Theory of Computing, STOC '74, pp. 47-63. ACM, New York, NY, USA (1974)

16. Ge, Y., Barrett, C., Tinelli, C.: Solving quantified verification conditions using satisfiability modulo theories. Annals of Mathematics and Artificial Intelligence 55(1-2), 101-122 (2009)

17. Ge, Y., de Moura, L.: Complete instantiation for quantified formulas in satisfiability modulo theories. In: Proceedings of CAV'09, LNCS, vol. 5643, pp. 306-320. Springer (2009)

18. Goel, A., Krstić, S., Leslie, R., Tuttle, M.: SMT-based system verification with DVF. In: Proceedings of SMT'12 (2012)

19. Ihlemann, C., Jacobs, S., Sofronie-Stokkermans, V.: On local reasoning in verification. In: C.R. Ramakrishnan, J. Rehof (eds.) TACAS 2008, pp. 265-281. Springer Berlin Heidelberg, Berlin, Heidelberg (2008)

20. Jovanovic, D., Barrett, C.: Being careful about theory combination. Formal Methods in System Design 42(1), 67-90 (2013)

21. Korovin, K.: iProver - an instantiation-based theorem prover for first-order logic. In: Proceedings of IJCAR'08, LNCS, vol. 5195, pp. 292-298. Springer (2008)

22. Kovács, L., Voronkov, A.: First-order theorem proving and vampire. In: Computer Aided Verification - 25th International Conference, CAV 2013, Saint Petersburg, Russia, July 13-19, 2013. Proceedings, pp. 1-35 (2013)

23. Krstić, S., Goel, A.: Architecting solvers for SAT modulo theories: Nelson-Oppen with DPLL. In: Proceeding of FroCoS'07, LNCS, vol. 4720, pp. 1-27. Springer (2007)

24. McCune, W.: A Davis-Putnam program and its application to finite first-order model search: Quasigroup existence problems. Tech. rep., Argonne National Laboratory (1994)

25. de Moura, L., Bjørner, N.: Efficient E-Matching for SMT solvers. In: Automated Deduction - CADE-21, 21st International Conference on Automated Deduction, Bremen, Germany, July 17-20, 2007, Proceedings, Lecture Notes in Computer Science, vol. 4603, pp. 183-198. Springer (2007)

26. Nieuwenhuis, R., Oliveras, A., Tinelli, C.: Solving SAT and SAT Modulo Theories: from an Abstract Davis-Putnam-Logemann-Loveland Procedure to DPLL(T). Journal of the ACM 53(6), 937-977 (2006)

27. Paulson, L.C., Wenzel, M.: Isabelle/HOL: a proof assistant for higher-order logic, vol. 2283. Springer (2002)

28. Reger, G., Suda, M., Voronkov, A.: Finding finite models in multi-sorted first-order logic. In: Theory and Applications of Satisfiability Testing - SAT 2016 - 19th International Conference, Bordeaux, France, July 5-8, 2016, Proceedings, pp. 323-341 (2016)

29. Reynolds, A., Tinelli, C., Goel, A., Krstić, S.: Finite model finding in SMT. In: N. Sharygina, H. Veith (eds.) Computer Aided Verification, Lecture Notes in Computer Science, vol. 8044, pp. 640-655. Springer Berlin Heidelberg (2013) 
30. Reynolds, A., Tinelli, C., Goel, A., Krstić, S., Deters, M., Barrett, C.: Quantifier instantiation techniques for finite model finding in SMT. In: M.P. Bonacina (ed.) Automated Deduction CADE-24, Lecture Notes in Computer Science, vol. 7898, pp. 377-391. Springer Berlin Heidelberg (2013)

31. Reynolds, A., Tinelli, C., de Moura, L.M.: Finding conflicting instances of quantified formulas in SMT. In: FMCAD, pp. 195-202. IEEE (2014). DOI 10.1109/FMCAD.2014. 6987613

32. Reynolds, A.J.: Finite model finding in satisfiability modulo theories. Ph.D. thesis, The University of Iowa (2013)

33. Schulz, S.: E-a brainiac theorem prover. Ai Communications 15(2, 3), 111-126 (2002)

34. Sutcliffe, G.: The TPTP Problem Library and Associated Infrastructure: The FOF and CNF Parts, v3.5.0. Journal of Automated Reasoning 43(4), 337-362 (2009)

35. Tinelli, C., Harandi, M.T.: A new correctness proof of the Nelson-Oppen combination procedure. In: Proceeding of FroCoS'96, Applied Logic, pp. 103-120. Kluwer Academic Publishers (1996)

36. Torlak, E., Jackson, D.: Kodkod: a relational model finder. In: Proceedings of TACAS'07, LNCS, vol. 4424, pp. 632-647. Springer (2007)

37. Tuttle, M.R., Goel, A.: Protocol proof checking simplified with SMT. In: Proceedings of NCA'12, pp. 195-202. IEEE Computer Society (2012)

38. Zhang, J., Zhang, H.: SEM: a system for enumerating models. In: Proceedings of IJCAI'95, pp. 298-303 (1995) 